\title{
Review on Experimental Research of Herbal Medicines with Anti-Amnesic Activity
}

Authors

Affiliations
Ming Tsuen Hsieh ${ }^{1}$, Wen Huang Peng ${ }^{1}$, Chi Rei Wu ${ }^{1}$, Kit Ying $\mathrm{Ng}^{2}$, Chuen Lung Cheng ${ }^{2}$, Hong Xi Xu ${ }^{2}$

${ }^{1}$ Graduate Institute of Chinese Pharmaceutical Sciences, College of Pharmacy, China Medical University, Taichung, Taiwan

${ }^{2}$ Hong Kong Jockey Club Institute of Chinese Medicine, Hong Kong, People's Republic of China
Key words

- amnesia

- herbal medicines

- cholinergic system

- GABAergic system

- glutaminergic system

- memory enhancement received August 21, 2009

revised October 7,2009

accepted Nov. 20, 2009

Bibliography

DOI http://dx.doi.org/

10.1055/s-0029-1240707

Published online December 23,

2009

Planta Med 2010; 76: 203-217

(c) Georg Thieme Verlag KG

Stuttgart · New York

ISSN 0032-0943

\section{Correspondence \\ Dr. Hong Xi Xu}

Hong Kong Jockey Club Institute

of Chinese Medicine

Unit 703, 7/F

Bio-Informatics Centre

Hong Kong Science Park

Shatin

Hong Kong

People's Republic of China

Phone: +85234062873

Fax: +85235517333

xuhongxi@hkjcicm.org

\section{Abstract}

$\nabla$

Amnesia is characterized by the inability to form memories or total or partial loss of memory secondary to cerebral malfunction following degenerative diseases, cerebral infections, traumatic injuries and emotional events which could be differentiated from dementia. However, no effective treatment for amnesia is currently available. Much research effort has been focused on developing new drugs from herbal medicines which have multifunctional properties. Novel plant extracts and their major or bioactive components including alkaloids, flavonoids, glycosides and saponins with promising antioxidant effects, various effects on cholinergic, GABAergic, glutaminergic, serotonergic, catecholaminergic and histaminergic systems, enhancement of cerebral blood flow and elevation of ribonucleic acid (RNA) as well as protein levels have been studied. In this review, we discuss the research findings on novel plant extracts and their bioactives with anti-amnesic effects on different neurotransmitter systems. Developing new drugs from herbal medicines for the treatment of amnesia is a hopeful attempt to meet the unmet medical needs.

\section{Abbreviations $\nabla$ \\ 5,7-DHT: 5,7-dihydrotroptamine \\ 5-HT: serotonin \\ 6-OHDA: 6-hydroxydopamine \\ ACh: acetylcholine}

AChE: acetylcholinesterase

AD: $\quad$ Alzheimer's disease

AGR: $\quad$ Acori graminei Soland., Araceae rhizome

AMPA: alpha-amino-3-hydroxy-5-methyl4-isoxazolepropionic acid

BBB: blood-brain barrier

BDPH: $\quad n$-butylidenephthalide

cDNA: complimentary deoxyribonucleic acid

ChAT choline-acetyltransferase

CNS: $\quad$ central nervous system

E-p-MCA: E-p-methoxycinnamic acid

FDA: $\quad$ Food and Drug Administration

GABA: gamma-aminobutyric acid

GBE: $\quad$ Ginkgo biloba L., Ginkgoaceae extract

GSH: glutathione

HBA: $\quad$-hydroxybenzyl alcohol

LDW: $\quad$ Liuwei Dihuang Wang

LTP: long-term potentiation

MAO: monoamine oxidase

MCA-Hg: 8-O-E-p-methoxycinnamoylharpagide

MDA: malondialdehyde

NMDA: $\quad N$-methyl-D-aspartic acid

PD/PT ratio: protopanaxadiol/protopanaxatriol ratio

RGTS: $\quad$ red ginseng total saponins

RNA: ribonucleic acid

RT-PCR: reverse transcriptase polymerase chain reaction

SOD: $\quad$ superoxide dismutase

TBARS: thiobarbituric acid reactive species

\section{Introduction}

$\nabla$

Amnesia is characterized by the inability to form memories or total or partial loss of memory secondary to cerebral malfunction following degenerative diseases such as Alzheimer's disease (AD), cerebral infections such as herpes or encephalitis, traumatic injuries such as stroke, alcohol or drug abuse and emotional events such as psychological trauma. The limbic system, comprised of the hippocampus, amygdala and parts of the cortex, is responsible for retrieving memory. Memory for- 
mation can be broadly categorized into three distinctive stages, namely learning acquisition, memory consolidation and retrieval. Despite the extensive causes of amnesia, the major affected brain regions are usually the subcortical region called the diencephalon and the cortical region called the medial temporal lobe [1]. The definition of amnesia can vary considerably according to different agreements on the term. This review focuses on recent literature on anterograde amnesia, which is the impaired ability to learn new information following the onset of amnesia, and retrograde amnesia, which is the impaired ability to recall past events and previously familiar information. Although amnesia is easily confused with dementia, they are distinguishable from each other. Unlike dementia, the amnesic memory loss does not affect a person's intelligence, general knowledge, awareness, attention span, judgment, personality or identity. Amnesia can happen to people at any age which can be a heavy burden to family members and the society, and requires extensive medical care and support schemes.

No conventional or alternative therapy is currently available to cure amnesia. Current therapeutic strategies for amnesia are mainly focused on enhancing or restoring cerebral circulation, restoring the level of neurotransmitters including acetylcholine (ACh), scavenging free radicals and restoring cell membrane fluidity. In the management of amnesia as well as AD, sustained treatment with cholinesterase inhibitors including donepezil, rivastigmine, galantamine and tacrine have been used. However, these drugs have questionable efficacy and may induce severe side effects including nausea, vomiting, diarrhea and muscle cramps.

Since ancient times, herbal medicines have been documented and used for nootropics (cognition-enhancing agents which aim at improving concentration, memory retention and problemsolving ability). In China, for example, the use of Panax ginseng C.A. Meyer (Araliaceae) to promote health and improve learning and memory is well documented [2-4]. Nowadays, Ginkgo biloba L. (Ginkgoaceae) extract (GBE) has been widely used as a health supplement to promote memory and standardized GBE has been approved in the German Commission E Monograph for the symptomatic treatment of the memory impairment, concentration difficulties and depression that result from organic brain disease [5]. In the past few years, the uses of herbal medicines for the treatment of cognitive impairments such as amnesia, AD or dementia have been widely studied; some drugs developed from herbs have shown prominent clinical improvements. A good example is galantamine (also known as galanthamine), which is an alkaloid extracted from the bulbs of Galanthus nivalis L. (Amaryllidaceae) with a promising effect for the treatment of $A D$ as a competitive reversible acetylcholinesterase (AChE) inhibitor [6]. It inhibits the hydrolysis of endogenous $\mathrm{ACh}$ and enhances the release of ACh by modulating nicotinic receptors in cholinergic system, thereby increasing the availability of ACh in the postsynaptic membrane. It is now one of the four cholinesterase inhibitors approved by the Food and Drug Administration (FDA) for the treatment of dementia [7]. In addition, galantamine is able to cross the blood-brain barrier (BBB) and therefore has high bioavailability.

Over the years, scientists have exerted great efforts to identify new anti-amnesic compounds from herbal medicines to meet the unmet medical needs. Potential new drugs from herbal medicines may be relevant in the treatment of cognitive disorders including amnesia. Herbal medicines can not only act synergistically with other components from the same herb, but also en- hance the activity of active components from other herbs in accordance with traditional practices including traditional Chinese and Ayurvedic medicines.

Herbal medicines may confer anti-amnesic protection by scavenging free radicals, enhancing cerebral blood flow, restoring ribonucleic acid (RNA) and protein levels. Besides, neurotransmitter systems were shown to play pivotal roles in cognitive processes where interactions between systems give rise to memory formation, consolidation and retrieval. Meanwhile, deficits in cholinergic, GABAergic, glutaminergic, serotonergic, catecholaminergic and histaminergic neurotransmissions can account at least partly for the pathophysiology of amnesia. Long-term potentiation is an important process in learning and memory, the action of which can be affected by changes in cholinergic, dopaminergic, noradrenergic and serotonergic systems [8-12]. Numerous research findings have revealed the involvement of different neurotransmitters in memory processes. Glutamate, GABA, dopamine and acetylcholine were shown to have stronger impacts on cognitive processes than serotonin and norepinephrine [13]. As reported in a number of experimental paradigms, administration of NMDA antagonists like MK-801and APV, AMPA antagonists like NBQX and CNQX, GABA $A_{A}$ agonists like diazepam and muscimol, GABA $A_{B}$ antagonist (CGP 464381) and agonist (baclofen), muscarinic antagonists like scopolamine and atropine, nicotinic antagonist like mecamylamine, serotonin $5-\mathrm{HT}_{1 \mathrm{~A}}$ agonist like alaproclate and buspirone, norepinephrine $\alpha-1$ antagonist like prazosin, $\alpha-2$ agonist like dexmedetomidine and a $\beta$ antagonist like propranolol, and dopamine D-2 antagonists like haloperidol and sulpiride led to memory impairment, while the uses of AMPA agonist like piperidine and norepinephrine $\alpha-1$ agonist like St-587 resulted in memory enhancement [13].

The literature was retrieved by searches of the most popular database Pubmed, using different combinations of keywords including "herbs", "herbal medicines", "amnesia", "memory enhancement”, "anti-oxidant”, “cholinergic”, “GABAergic”, "glutaminergic", "serotonergic", "catecholaminergic" and "histaminergic". The search covered the period from 1983 to March 2009. Reviews and research articles related to retrograde and anterograde amnesia were included. Additional papers of interest were retrieved from the reference lists of the above articles. We manually retrieved some recognized articles which are not available electronically. To construct a meaningful discussion on anti-amnesic effect of herbal medicine, studies totally without any attempt at mechanistic elucidation were excluded.

This paper summarizes the research findings and provides an overview of herbal medicines as treatments for both retrograde and anterograde amnesia. In this review, we present evidence for the potential benefit of herbal medicines in treating cognitive impairment following amnesia with special emphasis on actions through neurotransmitter systems. We first review the efficacies of various herbal medicines in in vivo and in vitro models of amnesia. Then, we discuss the characteristics of herbs which are suitable for treating amnesia, current issues and future perspectives for research and development of herbal medicines against amnesia.

\section{Chemical Agents Used to Induce Amnesia Models} $\nabla$

Various chemical agents have been frequently employed to induce amnesia in animal models to examine the anti-amnesic effects of the active ingredients from natural sources. In fact, these 
chemical agents possess different mechanistic actions on neural systems and intervene in the memory formation process at different stages. Scopolamine is a muscarinic acetylcholine receptor antagonist that induces memory deficits and is used widely to investigate cholinergic influence on learning ability in experimental animals. Scopolamine impairs learning acquisition that shortens the step-through latency in a passive avoidance task [14]. In the process of memory consolidation, new protein transcriptions and synthesis are needed [15]. Cycloheximide is an inhibitor of protein synthesis which impairs the memory consolidation process $[16,17]$. Apomorphine is used to impair the process of memory retrieval [18]. At high doses, it is a dopamine receptor agonist that increases dopaminergic activity and impairs memory retrieval in rodents. $p$-Chloroamphetamine is a serotonergic releaser which enhances serotonergic activity and is used to impair acquisition performance in animal models [19]. Mecamylamine is a nicotinic cholinergic antagonist. Blockade of the nicotinic receptor impairs learning acquisition [20]. Dizocilpine is also known as MK-801, a noncompetitive NMDA receptor antagonist which impairs memory reactivation [21]. Ibotenic acid impairs reference and working memory as revealed by poor performances in both spatial and associative radial maze tasks [22, 23]. It also provokes neuronal necrosis by overstimulation of $N$ methyl-D-aspartate (NMDA) receptors and elicits a sign of Alzheimer-type neurodegeneration [22]. Amnesia was induced by microinjection of ibotenic acid into the medial septal area. Ibotenic acid causes impairment of memory acquisition by decreasing ChAT activities in the hippocampus and frontal cortex [24].

\section{Mechanisms of Actions Concluded from Animal Studies $\nabla$}

Many herbs have been reported to improve memory and learning. They work through different mechanisms by affecting cholinergic, GABAergic, glutaminergic, serotonergic, histaminergic and catecholaminergic nervous systems. Natural products isolated from single herbs have been widely examined for their efficacies on amnesia. Through the isolation and structural determination, new drug candidates with promising anti-amnesic effects were identified. Interestingly, extracts of herbal formulations in traditional practice have also been studied and showed significant effects. These herbs and herbal formulae with anti-amnesic effects are introduced and have been listed in $\bullet$ Table 1.

\section{Action through cholinergic nervous system}

Cholinergic transmission in the brain cortical and hippocampal regions plays a fundamental role in memory [25]. Improved cholinergic neurotransmission can be achieved by increasing stimulation of cholinergic receptors and increasing the availability of ACh in the neuronal synaptic cleft. Muscarinic $\mathrm{M}_{2}$ autoreceptor inhibitors increase the release of ACh [26] while cholinesterase inhibitors decrease the breakdown of ACh. Cholinesterase inhibitors that block AChE and/or butyrylcholinesterase are the most common pharmacotherapy for amnesia, dementia and Alzheimer's disease. Several AChE inhibitors have been approved by the FDA for alleviation of symptoms; however, permanent improvement has not yet been achieved. The use of tacrine, the first licensed AChE inhibitor, is highly restricted due to its hepatotoxicity $[27,28]$.

Angelica acutiloba (Siebold \& Zucc.) Kitag. (Apiaceae) and Paeonia lactiflora Pall. (Paeoniaceae): A traditional formulation, Shimotsu-to, is composed of four herbs namely A. acutiloba (Japa- nese angelica root), $P$. lactiflora (peony root), Cnidium officinale Makino. (Umbelliferae) and Rehmannia glutinosa Libosch. (Scrophylariaceae). Peony root extract and Japanese angelica root extract have been shown to significantly attenuate scopolamine-induced amnesia in rats [29]. As indicated by the performance of paeoniflorin (1) ( Fig. 1) in treated rats in the eight arm radial maze, it can significantly attenuate the scopolamine-induced amnesia. Paeoniflorin helped recover the scopolamine-induced decline of ACh content in the striatum. However, active components of the other three herbs in this formulation were not resolved and their roles in memory-enhancing effect remain unclear.

Angelica gigas Nakai. (Umbelliferae): Decursin (2) († Fig. 1), a major coumarin constituent of A. gigas, significantly ameliorated scopolamine-induced amnesia in passive avoidance test and Morris water maze test. Decursin exerted its anti-amnesic activity in vivo in part by inhibiting AChE activity in the hippocampus [30]. Nodakenin (3) ( Fig. 1), a coumarin, is also isolated from the roots of A.gigas. Rats/mice treated with nodakenin antagonized the scopolamine-induced cognitive impairments in passive avoidance and Y-maze test. In the Morris water maze test, the escape latency during training was reduced whereas swimming times and distances within the target zone were increased in the nodakenin-treated amnesic group. Nodakenin exhibited an inhibitory effect on AChE activity in in vitro and ex vivo studies. It is suggested that nodakenin conferred beneficial effect against cognitive impairment through enhancement of cholinergic signaling [31]. In summary, decursin and nodakenin derived from A. gigas are two active ingredients with promising anti-amnesia activity. Of note, decursin showed a higher AChE inhibitory effect than the positive control velnacrine (a known AChE inhibitor). Decursin, a chemical derivative of decursinol which was present in the highest quantity in A.gigas, with the addition of an isoprenyl unit at C-6 of coumarin derivatives, facilitates its penetration through cellular membranes like BBB [32].

Acorus gramineus Soland. (Araceae): Acori graminei rhizoma (AGR) is the dried rhizomes of Acorus gramineus Solander (Araceae), which have been shown to have a protective effect against stroke, AD and vascular dementia [33-35]. AGR alleviated learning and memory deficits as revealed by the improved performance on the Morris Water maze. It conferred neuroprotection partly through attenuation of ibotenic acid-induced decrease of acetylcholinergic neurons in the hippocampus [36].

Angelica sinensis (Oliv.) Diels. (Apiaceae) and Cnidium officinale Makino. (Umbelliferae): $n$-Butylidenephthalide (BDPH) (4) (- Fig. 1) is an active lipophilic ingredient isolated from $A$. sinensis and $C$. officinale. BDPH attenuated scopolamine and/or mecamylamine-induced acquisition impairment. It was found that a peripheral cholinergic muscarinic receptor antagonist (scopolamine methylbromide) did not inhibit the counteracting effect of BDPH against scopolamine-induced acquisition impairment. BDPH attenuated the AF64A (central acetylcholinergic neurotoxin) induced cognitive impairment. These findings suggest that the cognitive enhancing effect of BDPH acted through the activation of the central muscarinic and nicotinic receptors but not the peripheral cholinergic neuronal systems [37].

Angelica sinensis (Oliv.) Diels. (Apiaceae) and Ligusticum wallichii Franch. (Apiaceae): Ferulic acid (5) ( Fig. 1) can be isolated from A. sinensis, L. wallichii and many other plants. Ferulic acid reversed the scopolamine- and cycloheximide-induced cognitive impairment but not the $p$-chloroamphetamine-induced impairment by activation of the cholinergic system and enhancement of brain microcirculation [38]. 
Table 1 Summary on anti-amnesic effects of herbal extracts.

\begin{tabular}{|c|c|c|c|c|}
\hline Name of herbs/formulae & $\begin{array}{l}\text { Active compound or fraction, } \\
\text { effective dose and treatment } \\
\text { duration }\end{array}$ & $\begin{array}{l}\text { Possible mechanisms on } \\
\text { nervous system }\end{array}$ & Types of models & Reference \\
\hline $\begin{array}{l}\text { Acorus gramineus Soland., } \\
\text { Araceae }\end{array}$ & $\begin{array}{l}\text { Methanol extract of Acori graminei } \\
\text { rhizome (AGR); } 100 \mathrm{mg} / \mathrm{kg} \text {, i.p.; } \\
21 \text { days }\end{array}$ & $\begin{array}{l}\text { Attenuated ibotenic acid-induced } \\
\text { decrease of acetylcholinergic } \\
\text { neuron in hippocampus }\end{array}$ & $\begin{array}{l}\text { Ibotenic acid ( } 4 \mu \mathrm{g} / \mu \mathrm{L} \text { artificial } \\
\text { CSF, microinjection into medial } \\
\text { septal area) induced in rats }\end{array}$ & [36] \\
\hline \multirow[t]{2}{*}{$\begin{array}{l}\text { Angelica gigas Nakai., } \\
\text { Umbelliferae }\end{array}$} & $\begin{array}{l}\text { Decursin; } 1 \text { and } 5 \mathrm{mg} / \mathrm{kg} \text {, i. p.; } \\
\text { single administration }\end{array}$ & $\begin{array}{l}\text { Inhibited AChE activity by } 34 \% \text { in } \\
\text { the hippocampus }\end{array}$ & $\begin{array}{l}\text { Scopolamine (1 mg/kg, i.p.) } \\
\text { induced in mice }\end{array}$ & [31] \\
\hline & $\begin{array}{l}\text { Nodakenin; } 10 \mathrm{mg} / \mathrm{kg}, \text { p. o.; single } \\
\text { administration }\end{array}$ & $\begin{array}{l}\text { Inhibited AChE activity in in vitro } \\
\text { study }\left(\mathrm{IC}_{50}=84.7 \mu \mathrm{M}\right) \text {; Inhibited } \\
\text { AChE activity for } 6 \mathrm{~h} \text { in ex vivo } \\
\text { study }\end{array}$ & $\begin{array}{l}\text { Scopolamine }(1 \mathrm{mg} / \mathrm{kg} \text {, i.p. }) \\
\text { induced in mice }\end{array}$ & [32] \\
\hline $\begin{array}{l}\text { Angelica sinensis (Oliv.) Diels., } \\
\text { Apiaceae } \\
\text { Cnidium officinale Makino., } \\
\text { Umbelliferae }\end{array}$ & $\begin{array}{l}\text { n-Butylidenephthalide (BDPH); } \\
50 \text { or } 100 \mathrm{mg} / \mathrm{kg} \text {, i.p.; single } \\
\text { administration }\end{array}$ & $\begin{array}{l}\text { By activation of central (via mus- } \\
\text { carinic and nicotinic receptors) } \\
\text { but not the peripheral cholinergic } \\
\text { neuronal systems }\end{array}$ & $\begin{array}{l}\text { Scopolamine }(1.0 \mathrm{mg} / \mathrm{kg} \text {, i.p.) } \\
\text { or mecamylamine }(10.0 \mathrm{mg} / \mathrm{kg} \text {, } \\
\text { i. p.) or scopolamine }(0.3 \mathrm{mg} / \\
\mathrm{kg} \text {, i. p.) plus mecamylamine } \\
(3 \mathrm{mg} / \mathrm{kg}, \text { i.p. }) \text { induced in rats }\end{array}$ & [37] \\
\hline $\begin{array}{l}\text { Angelica sinensis (Oliv.) Diels., } \\
\text { Apiaceae } \\
\text { Ligusticum wallichii Franch., } \\
\text { Apiaceae }\end{array}$ & $\begin{array}{l}\text { Ferulic acid; } 50 \text { and } 100 \mathrm{mg} / \mathrm{kg} \text {, } \\
\text { i. p.; single administration }\end{array}$ & $\begin{array}{l}\text { By activation of the cholinergic } \\
\text { system and enhancement of } \\
\text { brain microcirculation }\end{array}$ & $\begin{array}{l}\text { Scopolamine }(1.0 \mathrm{mg} / \mathrm{kg}, \text { i.p. }) \\
\text { or cycloheximide }(1.5 \mathrm{mg} / \mathrm{kg} \text {, } \\
\text { s. c.) induced in rats }\end{array}$ & [38] \\
\hline $\begin{array}{l}\text { Bacopa monniera (L.) Penn.syn., } \\
\text { Scrophulariaceae }\end{array}$ & $\begin{array}{l}\text { Standardized extract containing } \\
55.35 \% \text { bacosides; } 120 \mathrm{mg} / \mathrm{kg} \text {, } \\
\text { p. o., } 60 \mathrm{~min} \text {, single administra- } \\
\text { tion }\end{array}$ & $\begin{array}{l}\text { Act through GABA- benzodiaze- } \\
\text { pine pathway }\end{array}$ & $\begin{array}{l}\text { Diazepam }(1.75 \mathrm{mg} / \mathrm{kg} \text {, i. p. }) \text { in- } \\
\text { duced in mice }\end{array}$ & [70] \\
\hline $\begin{array}{l}\text { Coptis chinensis Franch., } \\
\text { Ranunculaceae }\end{array}$ & $\begin{array}{l}\text { Berberine; } 0.1 \text { or } 0.5 \mathrm{~g} / \mathrm{kg}, \text { p. o.; } \\
\text { 7-day or } 14 \text {-day }\end{array}$ & $\begin{array}{l}\text { Enhanced peripheral and central } \\
\text { cholinergic neuronal system } \\
\text { activities }\end{array}$ & $\begin{array}{l}\text { Scopolamine }(1.0 \mathrm{mg} / \mathrm{kg} \text {, i.p. }) \\
\text { induced in rats }\end{array}$ & [39] \\
\hline $\begin{array}{l}\text { Corydalis yanhusuo W.T. Wang., } \\
\text { Papaveraceae }\end{array}$ & $\begin{array}{l}\text { Pseudocoptisine; } 2.0 \mathrm{mg} / \mathrm{kg}, \text { p. o.; } \\
0.5,1,3,6 \text { or } 12 \text { hours }\end{array}$ & Inhibited AChE activity & $\begin{array}{l}\text { Scopolamine }(1.0 \mathrm{mg} / \mathrm{kg} \text {, i. p.) } \\
\text { induced in mice }\end{array}$ & [40] \\
\hline \multirow[t]{3}{*}{$\begin{array}{l}\text { Cnidium monnieri (L.) Cuss., } \\
\text { Apiaceae }\end{array}$} & $\begin{array}{l}\text { Osthole; } 3 \text { or } 10 \mathrm{mg} / \mathrm{kg} \text {, s. c.; } \\
3 \text { days in rats }\end{array}$ & $\begin{array}{l}\text { Mediated in part by activation of } \\
\text { the central cholinergic neuronal } \\
\text { system }\end{array}$ & $\begin{array}{l}\text { Scopolamine }(0.5 \mathrm{mg} / \mathrm{kg} \text {, i. p. }) \\
\text { induced in rats }\end{array}$ & [41] \\
\hline & $\begin{array}{l}10 \text { and } 30 \mu \mathrm{g} / \text { brain, icv; } 3 \text { days in } \\
\text { intact female rats }\end{array}$ & & & \\
\hline & $\begin{array}{l}30 \mu \mathrm{g} / \mathrm{brain}, i c v ; 3 \text { days in OVA } \\
\text { rats }\end{array}$ & & & \\
\hline $\begin{array}{l}\text { Desmodium gangeticum (L.) DC., } \\
\text { Fabaceae }\end{array}$ & $\begin{array}{l}\text { Aqueous extract; } 50,100 \text { or } \\
200 \mathrm{mg} / \mathrm{kg}, \text { p. o., } 7 \text { days }\end{array}$ & Decreased AChE activity & $\begin{array}{l}\text { Scopolamine }(0.4 \mathrm{mg} / \mathrm{kg} \text {, i.p. }) \\
\text { induced in mice }\end{array}$ & [42] \\
\hline Foeniculum vulgare Mill., Apiaceae & $\begin{array}{l}\text { Methanolic extract of the whole } \\
\text { plant; } 8 \text { days }\end{array}$ & Inhibited AChE activity & $\begin{array}{l}\text { Scopolamine }(0.4 \mathrm{mg} / \mathrm{kg} \text {, i.p.) } \\
\text { induced in mice }\end{array}$ & [43] \\
\hline Gastrodia elata BI., Orchidaceae & $\begin{array}{l}\text { p-Hydroxybenzyl alcohol (HBA); } \\
1,5 \text { or } 25 \mathrm{mg} / \mathrm{kg}, \text { p. o.; single ad- } \\
\text { ministration }\end{array}$ & $\begin{array}{l}\text { Acted through suppressing } \\
\text { dopaminergic and serotonergic } \\
\text { activities }\end{array}$ & $\begin{array}{l}\text { Scopolamine }(1.0 \mathrm{mg} / \mathrm{kg} \text {, i. p. }) \\
\text { induced in rats }\end{array}$ & {$[79,80]$} \\
\hline $\begin{array}{l}\text { Geissospermum vellosii Allem., } \\
\text { Apocynaceae }\end{array}$ & $\begin{array}{l}\text { Ethanolic extract of G. vellosi } \\
\text { stembarks; } 30 \mathrm{mg} / \mathrm{kg} \text {, i.p. } 45 \mathrm{~min}\end{array}$ & $\begin{array}{l}\text { Imposed anticholinesterase } \\
\text { activity }\end{array}$ & $\begin{array}{l}\text { Scopolamine }(1.0 \mathrm{mg} / \mathrm{kg} \text {, i. p. }) \\
\text { induced in mice }\end{array}$ & [44] \\
\hline \multirow[t]{3}{*}{ Ginkgo biloba L., Ginkgoaceae } & $\begin{array}{l}\text { Whole extract; } 30 \text { and } 60 \mathrm{mg} / \mathrm{kg} \text {; } \\
7 \text { consecutive days (once per day) }\end{array}$ & $\begin{array}{l}\text { Inhibited AChE activity in in vitro } \\
\text { study }\left({ }^{\prime} C_{50}=268.33 \mu \mathrm{g}\right) \text {; }\end{array}$ & $\begin{array}{l}\text { Scopolamine }(3.0 \mathrm{mg} / \mathrm{kg} \text {, i.p.) } \\
\text { induced in mice }\end{array}$ & [45] \\
\hline & $\begin{array}{l}\text { Whole extract; } 40 \text { and } 90 \mathrm{mg} / \mathrm{kg} \text {, } \\
\text { oral, } 7 \text { days }\end{array}$ & $\begin{array}{l}\text { Acted through glutamatergic } \\
\text { system enhancement }\end{array}$ & $\begin{array}{l}\text { MK-801 }(0.06 \mathrm{mg} / \mathrm{kg} \text { for } 1 \mathrm{~h} \text { or } \\
0.1 \mathrm{mg} / \mathrm{kg} \text { for } 1 \text { day, i. p. }) \text { in- } \\
\text { duced in rats }\end{array}$ & [75] \\
\hline & $\begin{array}{l}\text { Ethanol extract; } 30 \mathrm{mg} / \mathrm{kg} \text {, oral, } \\
1 \text { hour }\end{array}$ & $\begin{array}{l}\text { Acted through cholinergic and } \\
\text { histaminergic system enhance- } \\
\text { ment }\end{array}$ & $\begin{array}{l}\text { Scopolamine }(0.5 \mathrm{mg} / \mathrm{kg} \text {, i. p. }) \\
\text { induced in rats }\end{array}$ & [84] \\
\hline $\begin{array}{l}\text { Hypericum perforatum L., } \\
\text { Guttiferae }\end{array}$ & $\begin{array}{l}\text { Ethanolic extract; 4.0, 8.0, } 12.0 \text {, } \\
\text { and } 25.0 \mathrm{mg} / \mathrm{kg}, \text { i. } p . ; 1 \text { day, single } \\
\text { administration }\end{array}$ & $\begin{array}{l}\text { Acted through adrenergic and } \\
\text { serotonergic 5-HT1A receptors }\end{array}$ & $\begin{array}{l}\text { Scopolamine ( } 3.0 \mathrm{mg} / \mathrm{kg} \text {, i.p.) } \\
\text { induced in mice }\end{array}$ & [82] \\
\hline \multirow[t]{4}{*}{ Liuwei Dihuang Wang } & $\begin{array}{l}\text { Aqueous or ethanol extract; } 1 \text { or } \\
2 \mathrm{~g} / \mathrm{kg} \text {, p. o.; single administration }\end{array}$ & $\begin{array}{l}\text { By activating peripheral choliner- } \\
\text { gic neuronal system and modu- } \\
\text { lating the central nervous system }\end{array}$ & $\begin{array}{l}\text { Cycloheximide }(1.5 \mathrm{mg} / \mathrm{kg}, \text { s. c.) } \\
\text { induced in rats }\end{array}$ & [47] \\
\hline & $\begin{array}{l}\text { Aqueous extract; } 1 \mathrm{~g} / \mathrm{kg} \text { for } 7 \\
\text { days or } 0.1 \mathrm{~g} / \mathrm{kg} \text { for } 14 \text { days, p.o. }\end{array}$ & & & \\
\hline & $\begin{array}{l}\text { Aqueous extract; } 1 \mathrm{~g} / \mathrm{kg} \text { for } \\
7 \text { days; } 0.01 \mathrm{~g} / \mathrm{kg} \text { for } 14 \text { days }\end{array}$ & not mentioned & $\begin{array}{l}\text { Scopolamine }(1.0 \mathrm{mg} / \mathrm{kg} \text {, i.p. }) \\
\text { induced in rats }\end{array}$ & [48] \\
\hline & $\begin{array}{l}\text { Aqueous extract; } 0.1-1.0 \mathrm{~g} / \mathrm{kg} \text { for } \\
7 \text { days; } 0.01 \mathrm{~g} / \mathrm{kg} \text { for } 14 \text { days }\end{array}$ & not mentioned & $\begin{array}{l}\text { p-Chloroamphetamine } \\
(2.5 \mathrm{mg} / \mathrm{kg}) \text { induced in rats }\end{array}$ & $\begin{array}{l}{[48]} \\
\text { (continued) }\end{array}$ \\
\hline
\end{tabular}


Table 1 (continued)

\begin{tabular}{|c|c|c|c|c|}
\hline Name of herbs/formulae & $\begin{array}{l}\text { Active compound or fraction, } \\
\text { effective dose and treatment } \\
\text { duration }\end{array}$ & $\begin{array}{l}\text { Possible mechanisms on } \\
\text { nervous system }\end{array}$ & Types of models & Reference \\
\hline \multirow[t]{2}{*}{$\begin{array}{l}\text { Lonicera japonica Thunb., } \\
\text { Caprifoliaceae }\end{array}$} & $\begin{array}{l}\text { Luteolin; } 5 \text { or } 10 \mathrm{mg} / \mathrm{kg} \text {, i. p.; } \\
\text { single administration }\end{array}$ & $\begin{array}{l}\text { Enhanced activities of central } \\
\text { muscarinic and nicotinic recep- } \\
\text { tors }\end{array}$ & $\begin{array}{l}\text { Scopolamine }(0.5 \mathrm{mg} / \mathrm{kg} \text {, i. p. }) \\
\text { induced in rats }\end{array}$ & [49] \\
\hline & $\begin{array}{l}\text { Luteolin; } 5 \text { or } 10 \mathrm{mg} / \mathrm{kg}, \text { p. o.; } \\
\text { single administration }\end{array}$ & $\begin{array}{l}\text { Modulated microvascular func- } \\
\text { tion; Increased in regional cere- } \\
\text { bral blood flow; Efficient clear- } \\
\text { ance of reactive oxygen species; } \\
\text { Restored ACh level and reduced } \\
\text { AChE activity; Increased in brain- } \\
\text { derived neurotrophic factor level } \\
\text { and its receptor tyrosine kinase B } \\
\text { expression in cerebral cortex }\end{array}$ & $\begin{array}{l}\text { Amyloid } \beta \text { (aggregated form, } \\
3 \mu \mathrm{l}, \mathrm{icv} \text { ) induced in mice }\end{array}$ & {$[50]$} \\
\hline $\begin{array}{l}\text { Murraya koenigii (L.) Roxb., } \\
\text { Rutaceae }\end{array}$ & $\begin{array}{l}\text { Leaves of M. koenigii (grinded into } \\
\text { powder), } 2,4 \text { and } 8 \% \text { w/w, p. o.; } \\
\text { single dose for } 30 \text { days }\end{array}$ & $\begin{array}{l}\text { Inhibited brain cholinesterase } \\
\text { activity }\end{array}$ & $\begin{array}{l}\text { Scopolamine }(0.4 \mathrm{mg} / \mathrm{kg} \text {, i. p. }) \\
\text { induced in mice }\end{array}$ & {$[51]$} \\
\hline $\begin{array}{l}\text { Nardostachys jatamansi DC., } \\
\text { Valerianaceae }\end{array}$ & $\begin{array}{l}\text { Ethanolic extract; } 200 \mathrm{mg} / \mathrm{kg} \text {, } \\
\text { p. o.; } 8 \text { days }\end{array}$ & $\begin{array}{l}\text { Facilitated cholinergic transmis- } \\
\text { sion and acted through antioxida- } \\
\text { tion }\end{array}$ & $\begin{array}{l}\text { Scopolamine }(0.4 \mathrm{mg} / \mathrm{kg}, \text { i. p. }) \\
\text { or diazepam }(1.0 \mathrm{mg} / \mathrm{kg} \text {, i.p.) }\end{array}$ & [52] \\
\hline $\begin{array}{l}\text { Nelumbo nucifera Gaertn., } \\
\text { Nymphacaceae }\end{array}$ & $\begin{array}{l}\text { Lyophilized aqueous extract of } \\
N \text {. nucifera semen; } 1 \mathrm{~g} / \mathrm{kg} \text {, i.p.; } \\
\text { single administration }\end{array}$ & $\begin{array}{l}\text { Inhibited ACHE activity and in- } \\
\text { creased CHAT expression }\end{array}$ & $\begin{array}{l}\text { Scopolamine }(1 \mathrm{mg} / \mathrm{kg} \text {, i.p.) } \\
\text { induced in rats }\end{array}$ & [53] \\
\hline \multirow[t]{2}{*}{$\begin{array}{l}\text { Paeonia lactiflora Pall., } \\
\text { Paeoniaceae }\end{array}$} & $\begin{array}{l}\text { Whole extract of peony root; } \\
0.25 \text { and } 1 \mathrm{~g} \text { dried herb } / \mathrm{kg}, \text { p. o. }\end{array}$ & $\begin{array}{l}\text { Reversed scopolamine-induced } \\
\text { decrease in ACh content in } \\
\text { striatum }\end{array}$ & $\begin{array}{l}\text { Scopolamine }(0.3 \mathrm{mg} / \mathrm{kg} \text {, i. p. }) \\
\text { induced in rats }\end{array}$ & [29] \\
\hline & $\begin{array}{l}\text { Paeoniflorin; } 1 \text { mg/kg, p.o. } \\
\text { single administration }\end{array}$ & & & \\
\hline \multirow[t]{5}{*}{$\begin{array}{l}\text { Panax ginseng C. A. Mey., } \\
\text { Araliaceae }\end{array}$} & $\begin{array}{l}\text { Red ginseng total saponins; } \\
\text { single: } 200 \mathrm{mg} / \mathrm{kg}, \text { p. o.; or } \\
\text { repeated: } 200 \mathrm{mg} / \mathrm{kg} \text {, p. o.; } \\
7 \text { days }\end{array}$ & $\begin{array}{l}\text { Acted through catecholaminer- } \\
\text { gic neuronal system }\end{array}$ & $\begin{array}{l}\text { Ethanol }(3 \mathrm{~g} / \mathrm{kg}, \text { p. o. }) \text { induced in } \\
\text { rats }\end{array}$ & [87] \\
\hline & Rb1 & $\begin{array}{l}\text { Enhanced level of ACh in the CNS } \\
\text { (through increasing acetyltrans- } \\
\text { ferase activity or inhibiting AChE } \\
\text { activity); } \\
\text { Increased protein biosynthesis }\end{array}$ & & [57] \\
\hline & $\operatorname{Rg} 1$ & $\begin{array}{l}\text { Enhanced level of ACh in the CNS } \\
\text { (through increasing acetyltrans- } \\
\text { ferase activity or inhibiting AChE } \\
\text { activity); } \\
\text { Increased protein biosynthesis }\end{array}$ & & [57] \\
\hline & $\begin{array}{l}\mathrm{Rg} 3(\mathrm{R}), \mathrm{Rg} 3(\mathrm{~S}) \text { and } \mathrm{Rg} 5 / \mathrm{Rk} 1 ; \\
10 \mathrm{mg} / \mathrm{kg}, \text { p. o.; once a day for } \\
4 \text { days }\end{array}$ & $\begin{array}{l}\text { Conferred neuroprotection partly } \\
\text { through anti-excitotoxic abilities }\end{array}$ & $\begin{array}{l}\text { Ethanol }(3 \mathrm{~g} / \mathrm{kg}, \text { p. o. }) \text { induced in } \\
\text { mice }\end{array}$ & [76] \\
\hline & $\begin{array}{l}\mathrm{Rg} 3(\mathrm{~S}) \text { and } \mathrm{Rg} 5 / \mathrm{Rk} 1 ; 10 \mathrm{mg} / \mathrm{kg} \text {, } \\
\text { p. o.; once a day for } 4 \text { days }\end{array}$ & $\begin{array}{l}\text { Conferred neuroprotection partly } \\
\text { through anti-excitotoxic abilities }\end{array}$ & $\begin{array}{l}\text { Scopolamine ( } 3 \mathrm{mg} / \mathrm{kg} \text {, i.p.) } \\
\text { induced in mice }\end{array}$ & [76] \\
\hline \multirow[t]{2}{*}{$\begin{array}{l}\text { Polygala tenuifolia Willd., } \\
\text { Polygalaceae }\end{array}$} & $\begin{array}{l}\text { Methanolic extract }(50 \mathrm{mg} / \mathrm{kg}) \text {, } \\
\text { Acylated oligosaccharide-con- } \\
\text { taining fraction }(25 \mathrm{mg} / \mathrm{kg}), \text { p. o.; } \\
1 \text { day, single administration }\end{array}$ & $\begin{array}{l}\text { Enhanced central cholinergic } \\
\text { system }\end{array}$ & $\begin{array}{l}\text { Scopolamine }(0.2 \mathrm{mg} / \mathrm{kg}) \\
\text { induced in mice }\end{array}$ & [59] \\
\hline & $\begin{array}{l}\text { Tenuifoliside } B(3 \text { or } 10 \mathrm{mg} / \mathrm{kg} \text { ), } \\
\text { p. o.; } 1 \text { day, single administration }\end{array}$ & $\begin{array}{l}\text { Enhanced central cholinergic } \\
\text { system }\end{array}$ & $\begin{array}{l}\text { Scopolamine }(1.0 \mathrm{mg} / \mathrm{kg}) \\
\text { induced in rats }\end{array}$ & \\
\hline $\begin{array}{l}\text { Polygonatum multiflorum Thunb., } \\
\text { Polygonacease }\end{array}$ & $\begin{array}{l}\text { Emodin; } 3,15 \text { or } 30 \mathrm{mg} / \mathrm{kg}, \text { p. o.; } \\
\text { single administration }\end{array}$ & $\begin{array}{l}\text { The beneficial effects were am- } \\
\text { plified by serotonergic 5-HT } 1 \mathrm{~A} \\
\text { receptor partial agonist (8-OH- } \\
\text { DPAT) and 5- } \mathrm{HT}_{2} \text { receptor antag- } \\
\text { onist (ritanerin) but reduced by } \\
\text { muscarinic receptor antagonist } \\
\text { (scopolamine) }\end{array}$ & $\begin{array}{l}\text { Cycloheximide }(1.0 \mathrm{mg} / \mathrm{kg}, \text { s.c.) } \\
\text { induced in rats }\end{array}$ & [83] \\
\hline $\begin{array}{l}\text { Pueraria lobata (Willd.) Ohwi, } \\
\text { Fabaceae }\end{array}$ & $\begin{array}{l}\text { Puerarin; } 25-50 \mathrm{mg} / \mathrm{kg} \text {, i.p.; } \\
\text { single administration }\end{array}$ & $\begin{array}{l}\text { Enhanced cholinergic activity via } \\
\text { nicotinic receptors (but not mus- } \\
\text { carinic), activated NMDA recep- } \\
\text { tors and decreased serotonergic } \\
\text { neuronal activity }\end{array}$ & $\begin{array}{l}\text { Mecamylamine ( } 10 \mathrm{mg} / \mathrm{kg} \text {, } \\
\text { i.p.), p-chloroamphetamine } \\
\text { ( } 5 \mathrm{mg} / \mathrm{kg}, \text { i.p.) or dizocilpine } \\
\text { (i.e.MK-801, } 0.1 \mathrm{mg} / \mathrm{kg}, \text { i.p.) } \\
\text { induced in rats }\end{array}$ & $\begin{array}{l}{[60]} \\
\text { (continued) }\end{array}$ \\
\hline
\end{tabular}


Table 1 (continued)

\begin{tabular}{|c|c|c|c|c|}
\hline Name of herbs/formulae & $\begin{array}{l}\text { Active compound or fraction, } \\
\text { effective dose and treatment } \\
\text { duration }\end{array}$ & $\begin{array}{l}\text { Possible mechanisms on } \\
\text { nervous system }\end{array}$ & Types of models & Reference \\
\hline \multirow[t]{2}{*}{$\begin{array}{l}\text { Pueraria thunbergiana (Siebold. \& } \\
\text { Zucc.) Benth., Fabaceae }\end{array}$} & $\begin{array}{l}\text { Methanol extract of } P \text {. thunbergi- } \\
\text { ana; } 500 \mu \mathrm{g} / \mathrm{mL} \text { in assay mixture }\end{array}$ & $\begin{array}{l}\text { By activation effect ( } 46 \% \text { ) on ace- } \\
\text { tyltransferase in vitro; }\end{array}$ & $\begin{array}{l}\text { Scopolamine ( } 1.0 \mathrm{mg} / \mathrm{kg} \text {, s. c.) } \\
\text { induced in mice }\end{array}$ & {$[61]$} \\
\hline & $\begin{array}{l}\text { Daidzein; } 4.5 \mathrm{mg} / \mathrm{kg}, \text { p. o., } \\
4 \text { weeks }\end{array}$ & $\begin{array}{l}\text { By acting as a choline acetyltrans- } \\
\text { ferase activator for ACh biosyn- } \\
\text { thesis }\end{array}$ & & \\
\hline \multirow[t]{6}{*}{ Salvia miltiorrhiza Bge., Labiatae } & $\begin{array}{l}\text { Tanshinone I ( } 2 \text { or } 4 \mathrm{mg} / \mathrm{kg}, \text { p. o.); } \\
\text { single administration }\end{array}$ & $\begin{array}{l}\text { Slightly inhibited AChE activity in } \\
\text { vitro but not ex vivo }\end{array}$ & $\begin{array}{l}\text { Scopolamine ( } 1 \mathrm{mg} / \mathrm{kg} \text {, i. p.) } \\
\text { induced in mice }\end{array}$ & [62] \\
\hline & $\begin{array}{l}\text { Tanshinone I ( } 2 \mathrm{mg} / \mathrm{kg}, \text { p. o.); } \\
\text { single administration }\end{array}$ & $\begin{array}{l}\text { Posed GABA } / \text { benzodiazepine } \\
\text { receptor ligand property }\end{array}$ & $\begin{array}{l}\text { Diazepam }(1 \mathrm{mg} / \mathrm{kg} \text {, i. p.) } \\
\text { induced in mice }\end{array}$ & \\
\hline & $\begin{array}{l}\text { Tanshinone II A (10 or } 20 \mathrm{mg} / \mathrm{kg} \text {, } \\
\text { p. o.); single administration }\end{array}$ & $\begin{array}{l}\text { Posed } G A B A_{A} / \text { benzodiazepine } \\
\text { receptor ligand property }\end{array}$ & $\begin{array}{l}\text { Diazepam ( } 1 \mathrm{mg} / \mathrm{kg}, \text { i.p. }) \\
\text { induced in mice }\end{array}$ & [62] \\
\hline & $\begin{array}{l}\text { Tanshinone II A ( } 10 \text { or } 20 \mathrm{mg} / \mathrm{kg} \text {, } \\
\text { p. o.); single administration }\end{array}$ & $\begin{array}{l}\text { Slightly inhibited AChE activity in } \\
\text { vitro but not ex vivo }\end{array}$ & $\begin{array}{l}\text { Scopolamine ( } 1 \mathrm{mg} / \mathrm{kg} \text {, i.p.) } \\
\text { induced in mice }\end{array}$ & \\
\hline & $\begin{array}{l}\text { Cryptotanshinone }(10 \mathrm{mg} / \mathrm{kg} \text {, } \\
\text { p. o.); single administration }\end{array}$ & $\begin{array}{l}\text { Inhibited AChE activity for } 3 \mathrm{~h} \text { in } \\
\text { ex vivo study; Inhibitory effect on } \\
\left.\text { AChE in vitro (IC } \text { S }_{50} \text { value } 82 \mu \mathrm{M}\right)\end{array}$ & $\begin{array}{l}\text { Scopolamine (1 mg/kg, i.p.) } \\
\text { induced in mice }\end{array}$ & [62] \\
\hline & $\begin{array}{l}\text { 15,16-dihydrotanshinone I } \\
\text { ( } 2 \text { or } 4 \text { mg/kg, p. o.); single } \\
\text { administration }\end{array}$ & $\begin{array}{l}\text { Inhibited AChE activity for } 6 \mathrm{~h} \text { in } \\
\text { ex vivo study; Inhibitory effect on } \\
\left.\text { AChE in vitro (IC } \mathrm{C}_{50} \text { value } 25 \mu \mathrm{M}\right)\end{array}$ & $\begin{array}{l}\text { Scopolamine (1 mg/kg, i.p.) } \\
\text { induced in mice }\end{array}$ & {$[62]$} \\
\hline Salvia triloba L., Labiatae & $\begin{array}{l}\text { Hydroalcoholic extract; } 200 \text { or } \\
400 \mathrm{mg} / \mathrm{kg}), 1 \text { day, single admin- } \\
\text { istration }\end{array}$ & $\begin{array}{l}\text { Inhibited AChE activity; Posed } \\
\text { antioxidant activity }\end{array}$ & $\begin{array}{l}\text { Scopolamine }(1.0 \mathrm{mg} / \mathrm{kg}, \text { i. p. }) \\
\text { induced in mice }\end{array}$ & [63] \\
\hline \multirow[t]{2}{*}{$\begin{array}{l}\text { Schisandra chinensis (Turcz.) Baill., } \\
\text { Tremellaceae }\end{array}$} & $\begin{array}{l}\text { Gomisin } \mathrm{A} ; 5 \mathrm{mg} / \mathrm{kg}, \text { p. o.; single } \\
\text { administration }\end{array}$ & $\begin{array}{l}\text { Inhibited } A C h E \text { activity in in vitro } \\
\text { study }\left(\mathrm{IC}_{50}=15.5 \mu \mathrm{M}\right)\end{array}$ & $\begin{array}{l}\text { Scopolamine (1 mg/kg, i.p.) } \\
\text { induced in mice }\end{array}$ & [66] \\
\hline & $\begin{array}{l}\text { Water layer of fruit of S. chinensis; } \\
10 \text { and } 25 \text { mg/kg, p. o.; single ad- } \\
\text { ministration }\end{array}$ & $\begin{array}{l}\text { The ameliorating effect was am- } \\
\text { plified by treatment with seroto- } \\
\text { nergic } 5-\mathrm{HT}_{2} \text { receptor anatagon- } \\
\text { ist (ritanserin) }\end{array}$ & $\begin{array}{l}\text { Cycloheximide }(1.5 \mathrm{mg} / \mathrm{kg} \text {, s. c.) } \\
\text { induced in rats }\end{array}$ & [79] \\
\hline \multirow[t]{2}{*}{$\begin{array}{l}\text { Scrophularia buergeriana Miquel., } \\
\text { Scrophulariaceae }\end{array}$} & $\begin{array}{l}\text { (1) E-harpagoside; } 2 \text { mg/kg, p.o.; } \\
\text { single administration }\end{array}$ & $\begin{array}{l}\text { Increased glutathione reductase } \\
\text { and SOD as well as decreased GSH } \\
\text { and TBARS activities or contents; }\end{array}$ & $\begin{array}{l}\text { Scopolamine (1 mg/kg, i.p.) } \\
\text { induced in mice }\end{array}$ & [64] \\
\hline & $\begin{array}{l}\text { (2)8-O-E-p-methoxycinna moyl- } \\
\text { harpagide (MCA-Hg); } 2 \mathrm{mg} / \mathrm{kg} \text {, } \\
\text { p. o.; single administration }\end{array}$ & $\begin{array}{l}\text { Inhibited activity of AChE within } \\
\text { the cortex and hippocampus }\end{array}$ & & \\
\hline $\begin{array}{l}\text { Scutellaria baicalensis Georgi., } \\
\text { Lamiaceae }\end{array}$ & $\begin{array}{l}\text { Oroxylin } \mathrm{A}(5 \mathrm{mg} / \mathrm{kg}) \\
\text { single administration }\end{array}$ & $\begin{array}{l}\text { Acted through GABAergic } \\
\text { nervous system }\end{array}$ & $\begin{array}{l}\text { Scopolamine }(1 \mathrm{mg} / \mathrm{kg}, \text { i.p. }) \\
\text { induced in mice; or Diazepam } \\
(1 \mathrm{mg} / \mathrm{kg}, \text { i.p. }) \text { induced in mice }\end{array}$ & [71] \\
\hline Teucrium polium L., Lamiaceae & $\begin{array}{l}\text { Hydroalcoholic extract; } 200 \text { or } \\
400 \mathrm{mg} / \mathrm{kg}) ; 1 \text { day, single admin- } \\
\text { istration }\end{array}$ & $\begin{array}{l}\text { Inhibited AChE activity; } \\
\text { Posed antioxidant activity }\end{array}$ & $\begin{array}{l}\text { Scopolamine }(1.0 \mathrm{mg} / \mathrm{kg}, \text { i. p. }) \\
\text { induced in mice }\end{array}$ & [63] \\
\hline $\begin{array}{l}\text { Thespesia populnea Milo., } \\
\text { Malvaceae }\end{array}$ & $\begin{array}{l}\text { Ethanolic extract; } 200 \text { or } 400 \mathrm{mg} / \\
\mathrm{kg}, \text { p. o.; } 7 \text { days }\end{array}$ & $\begin{array}{l}\text { Reduced anticholinesterase } \\
\text { activity }\end{array}$ & $\begin{array}{l}\text { Scopolamine }(0.4 \mathrm{mg} / \mathrm{kg}, \text { i. p. }) \\
\text { induced in mice }\end{array}$ & {$[67]$} \\
\hline \multirow[t]{2}{*}{$\begin{array}{l}\text { Tremella fuciformis Berk., } \\
\text { Tremellaceae }\end{array}$} & $\begin{array}{l}\text { Aqueous extract; } 100 \text { or } 400 \mathrm{mg} / \\
\text { kg, p. o.; } 14 \text { days }\end{array}$ & $\begin{array}{l}\text { Acted through cholinergic } \\
\text { nervous system }\end{array}$ & $\begin{array}{l}\text { Scopolamine ( } 2 \mathrm{mg} / \mathrm{kg} \text {, s. c.) } \\
\text { induced in rats }\end{array}$ & [68] \\
\hline & $\begin{array}{l}\text { Aqueous extract; } 0.01,0.05,0.1 \\
\text { and } 1 \mu \mathrm{g} / \mathrm{mL}\end{array}$ & $\begin{array}{l}\text { Promoted neurite outgrowth of } \\
\text { PC12h cells }\end{array}$ & & \\
\hline $\begin{array}{l}\text { Uncariae ramulus et Uncus., } \\
\text { Rubiaceae }\end{array}$ & $\begin{array}{l}\text { Methanolic extract; } 100 \mathrm{mg} / \mathrm{kg} \text {, } \\
\text { i.p.; } 21 \text { days }\end{array}$ & $\begin{array}{l}\text { Reduced the loss of cholinergic } \\
\text { immunoreactivity in the hippo- } \\
\text { campus }\end{array}$ & $\begin{array}{l}\text { Ibotenic acid }(0.1 \mu \mathrm{L} \text { at concen- } \\
\text { tration } 4 \mu \mathrm{g} / \mu \mathrm{L}, i c v) \text { induced in } \\
\text { rat }\end{array}$ & [69] \\
\hline $\begin{array}{l}\text { Uncariae rhynchophylla (Miq.) } \\
\text { Jacks., Rubiaceae }\end{array}$ & $\begin{array}{l}\text { Methanolic extract }(250 \mathrm{mg} / \mathrm{kg} \text { ) } \\
\text { or alkaloid fraction ( } 25 \text { or } 50 \mathrm{mg} / \\
\mathrm{kg}), \text { p. o.; } 7 \text { days }\end{array}$ & $\begin{array}{l}\text { Restored the decrease in glutamic } \\
\text { acid and 5-hydroxytryptamine }\end{array}$ & $\begin{array}{l}\text { Ethanol [3 g/kg at concentra- } \\
\text { tion } 38 \%(v / v)] \text { induced in mice }\end{array}$ & [77] \\
\hline
\end{tabular}

Coptis chinensis Franch. (Ranunculaceae): Berberine (6) ( Fig. 1), isolated from C. chinensis, was shown to attenuate scopolamine-induced amnesia significantly. The beneficial effect was suggested to be linked to the increase in peripheral and central cholinergic neuronal system activities [39].

Corydalis yanhusuo W.T. Wang. (Papaveraceae): Pseudocoptisine is a quaternary benzylisoquinoline alkaloid isolated from the tuber of $C$. yanhusuo. Pseudocoptisine conferred anti-amnesic activity against scopolamine-induced learning and memory impairments partially via inhibition of AChE activity in a dose-dependent manner [40]. It is postulated that the detected AChE inhibitory activity might be traced back to the presence of a benzylisoquinoline alkaloid.

Cnidium monnieri (L.) Cuss. (Apiaceae): Osthole (7) (๑ Fig. 1), isolated from $C$. monnieri, reversed the scopolamine-induced performance deficit in ordinary female or ovariectomized rats in 


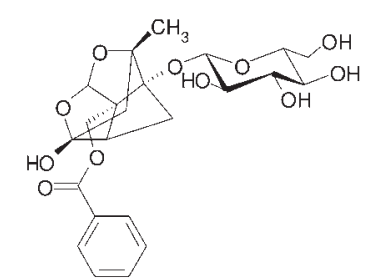

1<smiles>COc1cc(/C=C/C(=O)O)ccc1O</smiles>

5<smiles>O=c1cc(-c2ccc(O)c(O)c2)oc2cc(O)cc(O)c12</smiles>

9<smiles>C=CC1C=C2c3cc4c(cc3CC[N+]2=CC1C(=COC)OC)OCO4</smiles>

6

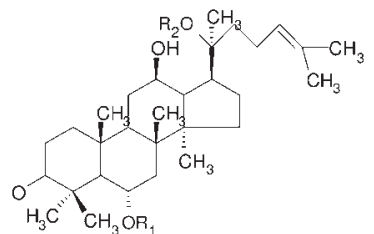
10

$\mathrm{R}_{1}=\mathrm{glc} ; \mathrm{R}_{2}=\mathrm{glc}$<smiles>O=c1c(-c2ccc(O)cc2)coc2cc(O)ccc12</smiles>

13<smiles>CC1CCC2=C1C(O)C(=O)c1c2ccc2ccccc12</smiles>

$\mathrm{CH}_{3}$

17<smiles>COc1c(O)cc2oc(-c3ccccc3)cc(=O)c2c1O</smiles>

21

Fig. 1 Chemical structures of paeoniflorin (1), decursin (2), nodakenin (3), butylidenephthalide (4), ferulic acid (5), berberine (6), osthole (7), (-)-huperzine A (8), n-luteolin (9), ginsenoside Rg1 (10), ginsenoside Rb1 (11), puerarin (12), daidzein (13), tanshinone I (14), tanshinone IIA (15), cryptotan-<smiles>CC1(C)OC(OC(O)C(O)CO)C1Cc1cc2ccc(=O)oc2cc1CO</smiles><smiles>CCC/C=C1\OC(=O)c2ccccc21</smiles>

4<smiles>COc1ccc2ccc(=O)oc2c1CC=C(C)C</smiles>

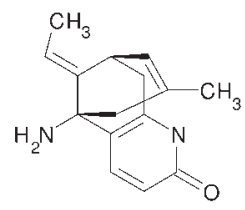

8<smiles>CC(C)=CCCC1CCC2(C)C(O)C1C(O)CC1C2CC(O)C2C(C)C(O)CCC12C</smiles>

11

$R_{1}=$ glc- - -glc; $R_{2}=$ glc -6 -glc<smiles>Cc1coc2c1C(=O)C(=O)c1c-2ccc2c1CCCC2(C)C</smiles>

15

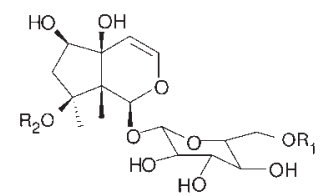

19

$\mathrm{R}_{1}=\mathrm{H}, \mathrm{R}_{2}=E-p$-methoxycinnamoyl<smiles>O=c1c(-c2ccc(O)cc2)coc2c(C(O)C(O)C(CO)C(O)CO)c(O)ccc12</smiles>

12<smiles>CC1COC2=C1C(=O)C(=O)c1c2ccc2c1CCCC2(C)C</smiles>

16

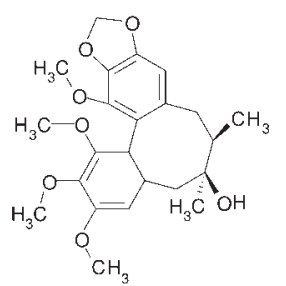

20<smiles>CC1=CC2C(=O)c3cc(O)cc(O)c3C(=O)C2C(O)=C1</smiles>

23

shinone (16), 15,16-dihydrotanshinone I (17), E-harpagoside (18), 8-O-E-pmethoxycinnamoylharpagide (19), gomisin A (20), oroxylin A (21), p-hydroxybenzyl alcohol (22) and emodin (23). part by mediating the activation of the central cholinergic neuronal system [41].

Desmodium gangeticum (L.) DC. (Fabaceae): Its aqueous extract reversed scopolamine induced amnesia by decreasing whole brain AChE activity [42].
Foeniculum vulgare Mill. (Apiaceae): A methanolic extract of the whole plant of $F$. vulgare ameliorated the amnesic effect of scopolamine by increasing step-down latency and inhibiting AChE activity [43]. 
Geissospermum vellosii Allem. (Apocynaceae): Pretreatment with an ethanolic extract of $G$. vellosi stem barks reduced scopolamine-induced amnesia as evidenced in passive avoidance and Morris water maze tests. G. vellosii showed potent anticholinersterase activity in vitro with a mean $\mathrm{IC}_{50}$ value of $39.3 \mu \mathrm{g} / \mathrm{mL}$ where geissospermine was identified as the main cholinesterase inhibitor [44].

Ginkgo biloba L. (Ginkgoaceae): Rats/mice pretreated with G. biloba extract resulted in significant inhibition of AChE activity in vivo and ex vivo [45]. It is postulated that the reduced AChE activity caused an increase in ACh level, which maintained learning and memory functions. The more pronounced anti-AChE activity was found in the detergent soluble fraction which mainly consists of the G4 form of the AChE of G. biloba, rather than the salt soluble fraction which mainly consists of the G1 isoform of the AChE. This implies that the G4 isoform of AChE is important in maintaining learning and memory functions.

Huperzia serrata (Thunb.) Trev. (Lycopodiaceae): (-)-Huperzine A (8) (๑ Fig. 1) is a natural Lycopodium alkaloid, extracted from $H$. serrata. Rats pretreated with (-)-huperzine A before scopolamine injection resulted in improvement of reference memory and working memory, as shown in radial maze performance. It was reported that (-)-huperzine A possessed unique anti-AChE activity [46].

Liuwei Dihuang Wang (LDW): LDW is a herbal formulation consisting of six herbs, namely Rhizoma of Rehmannia glutinosa Libosch. (Gesneriaceae), Fructus of Cornus officinalis Sieb. et Zucc. (Cornaceae), Radix of Dioscorea opposite Thunb. (Dioscoreaceae), Rhizoma of Alisma orientalis (Sam.) Juzep. (Alismataceae), Cortex of Paeonia suffruticosa Andr. (Paeoniaceae) and Poria cocos (Schw.) Wolf. (Polyporaceae). LDW ameliorated cycloheximide, scopolamine- or $p$-amphetamine-induced amnesia $[47,48]$. The ameliorating effects of LDW were blocked by a muscarinic antagonist (scopolamine), a peripheral cholinergic antagonist (scopolamine methylbromide), a serotonin precursor (5-hydroxytryptamien), a serotonin releaser (p-chloroamphetamine), a $\mathrm{GABA}_{\mathrm{A}}$ receptor antagonist (bicuculline) and $\mathrm{GABA}_{\mathrm{B}}$ receptor agonist (baclofen). These findings collectively implied that the ameliorating effects of LDW may be triggered by activating the peripheral cholinergic neuronal system and modulating the central nervous system.

Lonicera japonica Thunb. (Caprifoliaceae): Luteolin (9) (๑ Fig. 1) is usually found in celery, green pepper, perilla leaf and seed, chamomile tea and L. japonica. Luteolin reversed learning acquisition impairment induced by cholinergic neurotoxin, muscarinic (scopolamine hydrobromide) or nicotinic receptor antagonists. However, luteolin does not protect the brain from learning acquisition impairment induced by $\mathrm{N}$-methyl bromide, dopaminergic neurotoxin (6-hydroxydopamine, 6-OHDA) and serotonergic neurotoxin (5,7-dihydrotroptamine, 5,7-DHT). These findings suggested that luteolin protected the brain from scopolamine-induced learning acquisition impairment by enhancing the activities of central muscarinic and nicotinic receptors [49]. In another study, luteolin was shown to confer robust neurovascular protection in Abeta(25-35)-induced amnesia as evidenced by improvement in spatial learning and memory capabilities. This protection was attributed to a modulated microvascular function and increased regional cerebral blood flow, efficient clearance of reactive oxygen species, restored ACh level and reduced AChE activity, as well as increased brain-derived neurotrophic factor level and its receptor tyrosine kinase B expression in cerebral cortex [50].
Murraya koenigii (L.) Roxb. (Rutaceae): Leaves of M. koenigii were found to alleviate scopolamine-induced amnesia in young (3-4 months) and aged (12-15 months) mice. Inhibited brain cholinesterase activity was attributed to this protection [51].

Nardostachys jatamansi DC. (Valerianaceae): Its ethanolic extract has been shown to reverse scopolamine or diazepam-induced amnesia. This improvement can be attributed to facilitated cholinergic transmission [52] and its antioxidant property [52].

Nelumbo nucifera Gaertn. (Nymphacaceae): N. nucifera semen has been reported to have anti-diarrheal, anti-ganacratia, and tranquilizer-like activities. Lyophilized aqueous extract of $\mathrm{N}$. nucifera semen attenuated scopolamine-induced deficit in which the AChE activity of the N. nucifera treated group decreased to $7.35 \%$ and CHAT-positive neurons in the $N$. nucifera treated group increased by $51.02 \%$ compared with the control group. By inhibiting AChE activity and inducing CHAT expression, N. nucifera conferred anti-amnesic protection [53].

Panax ginseng C.A. Meyer (Araliaceae): Ginsenosides, the saponins of ginseng, are extracted from the root and rhizome of $P$. ginseng. The neuroprotective effects of ginsenosides have been widely studied in different models of neurological deficits like cerebral ischemia, Parkinson's disease and memory impairments. Ginsenoside Rg1 (10) ( Fig. 1) was proven to improve synaptic transmission and increase the amplitude of long-term potentiation $[54,55]$. It was revealed that ginsenosides $\mathrm{Rg} 1$ and Rb1 (11) (๑ Fig. 1) enhanced central nervous system (CNS) cholinergic metabolism. They were found to potentiate the cholinergic system by (1) increasing the density of central M-cholinergic receptors and (2) enhancing the level of ACh in the CNS (through increasing acetyltransferase activity or inhibiting AChE activity) $[56,57]$. Korean red ginseng saponins with a low PD/PT (protopanaxadiol/protopanaxatriol) ratio have been shown to improve spatial working memory whereas those with a high PD/PT ratio do not [45]. Ginsenoside Rg1, panaxatriol with two sugars, is generally more nootropic than Rb1, panaxadiol with four sugars. Increased protein biosynthesis as evidenced in the mouse brain may contribute to the memory consolidative effect conferred by ginsenosides Rb1 and Rg1 [58].

Polygala tenuifolia Willd. (Polygalaceae): It has been used for treatment of amnesia, neurasthenia, palpitation, noctural emission and insomnia. Either a methanolic extract or an acylated oligosaccharide-containing fraction ameliorated the scopolamineinduced decrease of retention in passive avoidance by enhancing the central cholinergic system [59].

Pueraria lobata (Willd.) Ohwi (Fabaceae): Puerarin (12) ( Fig. 1), isolated from P. lobata, has exhibited attenuation of mecamylamine-, $p$-chloroamphetamine- or dizocilpine-induced inhibitory avoidance performance deficits but not the scopolamine-induced one. The beneficial effect of puerarin might be attributed to the enhanced cholinergic activity via nicotinic but not muscarinic receptors as well as activated NMDA receptors and decreased serotonergic neuronal activity [60].

Pueraria thumbergiana (Siebold. \& Zucc.) Benth. (Fabaceae): Daidzein (13) ( Fig. 1) isolated from methanolic extracts of $P$. thunbergiana reversed the scopolamine-induced amnesia in the Y-maze test. Daidzein ameliorates scopolamine induced amnesia by acting as a choline acetyltransferase activator for Ach biosynthesis [61]. With extensive purification effort, daidzein has been successfully identified as the ChAT activator and one of the active ingredients responsible for memory enhancement. As admitted by the investigator, the precise working mechanisms for daidzein conferred memory enhancement remains largely unclear. Since 
only the cholinergic pathway was studied, it remains unknown whether daidzein conferred protection through a single direct action at the cholinergic system or a combinative effort occurring at multiple action sites.

Salvia miltiorrhiza Bge. (Labiatae): Tanshinones are a group of diterpenoids isolated from the roots of S. miltiorrhiza. Tanshinone I (14), tanshinone IIA (15), cryptotanshinone (16), and 15, 16-dihydrotanshinone I (17) ( $\bullet$ Fig. 1) are collectively called tanshinone congeners. Tanshinone I, tanshinone II A, cryptotanshinone and 15,16-dihydrotanshinone I significantly reversed the cognitive impairment induced by scopolamine. Cryptotanshinone and 15,16-dihydrotanshinone I were proven to induce an inhibitory effect on AChE in in vitro and ex vivo studies. Tanshinone congeners may exert a beneficial effect on cognitive impairment by cholinergic signaling enhancement [62].

Salvia triloba L. f. (Lamiaceae): Its hydroalcoholic extract exerted a memory enhancing effect partially through AChE inhibition with an $\mathrm{IC}_{50}$ value of $0.71 \mathrm{mg} / \mathrm{mL}$. Its hydroalcoholic extract exerted a memory enhancing effect partially through radical scavenging activity against 2,2-diphenylpicrylhydrazyl radical with an $\mathrm{IC}_{50}$ value of $0.227 \mathrm{mg} / \mathrm{mL}$ [63].

Scrophularia buergeriana Miq. (Scrophulariaceae): E-Harpagoside (18) and MCA-Hg (19) (๑ Fig. 1), isolated from S. buergeriana, enhance cognition by inhibiting the activity of AChE within the cortex and hippocampus to a level similar to that observed in donepezil-treated rats/mice [64]. In another study, E-p-methoxycinnamic acid (E-p-MCA), a phenylpropanoid isolated from roots of $S$. buergeriana, improved impairments of spatial learning and memory induced by scopolamine [65]. Although the underlying mechanism is not yet fully elucidated, the $\alpha, \beta$-unsaturated carboxyl moiety and the para-methoxy group in E-p-MCA are postulated to be crucial components in cognition-enhancing activity.

Schizandra chinesis (Turcz.) Baill. (Schisandraceae): Gomisin A (20) ( Fig. 1), an ingredient of fruits of S. chinesis, reversed the cognitive impairment induced by scopolamine in the passive avoidance test, Y-maze test and Morris water maze test. The cognition-enhancing effect of gomisin A was partially effected through inhibition of AChE activity dose-dependently [66].

Teucrium polium L. (Lamiaceae): Its hydroalcoholic extract exerted a memory enhancing effect partially through AChE inhibition with an $\mathrm{IC}_{50}$ value of $0.55 \mathrm{mg} / \mathrm{mL}$ [63].

Thespesia populnea Milo. (Malvaceae): It has been reported to have antifertility, antibacterial, anti-inflammatory, antioxidant, purgative and hepatoprotective activities. An ethanolic extract of $T$. populnea reversed the scopolamine-induced amnesia through reduced brain cholinesterase activity [67].

Tremella fuciformis Berk. (Tremellaceae): It has been demonstrated that T. fuciformis extract reduced scopolamine-induced learning and memory deficits by increasing the central cholinergic activity in the medial septum and hippocampus. In consistency with an animal study, the water extract of $T$. fuciformis promoted neurite outgrowth of PC12h cells. These findings have demonstrated that the anti-amnesic effect of $T$. fuciformis was conferred partly through the cholinergic system and promotion of neuritogenesis. Neurogenesis has been demonstrated to play a crucial role in regulating memory and learning; it would be helpful if the active ingredients responsible for the protection could be identified and further studied [68].

Uncariae ramulus et Uncus. (Rubiaceae): Its methanolic extract induced significant reversals of ibotenic acid-induced deficit in learning and memory by reducing the loss of cholinergic immunoreactivity in the hippocampus [69].

\section{Action through GABAergic nervous system}

Several studies have indicated the importance of GABAergic involvement in memory formation. Drugs enhancing $\mathrm{GABA}_{A}$ and $G_{B B A}$ neurotransmission cause memory impairment whereas drugs reducing $G_{A B A_{A}}$ neurotransmission lead to memory enhancement in rodents. Thus, modulation of GABAergic neurotransmission could possibly lead to advancements in treating amnesia.

Bacopa monniera (L.) Penn. syn. (Scrophulariaceae): B. monniera has been used for treating epilepsy, insomnia, anxiety and memory enhancement. Standardized extracts of B. monniera containing $55.35 \%$ bacosides have been shown to alleviate diazepam-induced amnesia as evidenced by decreased escape latency time. This protective effect may act through the GABA-benzodiazepine pathway possibly affecting long-term potentiation [70].

Salvia miltiorrhiza Bge. (Labiatae): Tanshinone I (14) and II A (15) ( Fig. 1), isolated from S. miltiorrhiza, restored the diazepam (a GABA $A$ /benzodiazepine receptor agonist) induced memory deficits in a passive avoidance test. This implied that tanshinone I or tanshinone II A prevented cholinergic dysfunction-related deterioration of learning and memory through the GABAergic neurotransmitter system [62].

Scutellaria baicalensis Georgi. (Lamiaceae): Oroxylin A (21) ( Fig. 1) is a flavonoid, isolated from the root of S. baicalensis. Rats/mice pretreated with oroxylin A significantly reversed the scopolamine or diazepam-induced cognitive impairments as revealed in passive avoidance and Y-maze testing. In the Morris water maze, the escape latencies in training trials were improved and the swimming times and distances within the target zone increased in oroxylin A treated amnesic rats/mice. The ameliorating effect of oroxylin A was reversed by the $\mathrm{GABA}_{\mathrm{A}}$ receptor agonists (either muscimol or diazepam). In addition, oroxylin $A$ inhibited the GABA-induced inward chloride ion current in a single cortical neuron. It was suggested that oroxylin A protected against scopolamine-induced memory impairment via the GABAergic nervous system [71].

\section{Action through glutamatergic nervous system}

Glutamate synapse is a potential target for drug intervention in amnesia. Two glutaminergic therapeutic approaches are AMPA and NMDA potentiations. Compounds like AMPAkines (potentiate glutamate's action at AMPA receptors) and D-cycloserine (a partial agonist at the glycine site of NMDA receptor) have demonstrated memory enhancement effects $[72,73]$. Glutamate receptors have been reported to play a pivotal role in learning and memory. Blockade of NMDA receptors with selective inhibitors abolishes long-term potentiation (LTP) in the CA1 region of the hippocampus and also blocks spatial leaning [74]. It is suggestive that NMDA receptors are involved in the learning process.

Ginkgo biloba L. (Ginkgoaceae): Chronic administration of G. biloba has been shown to remove memory disturbance induced by MK-801, which is a $N$-methyl-D-aspartic acid (NMDA) receptor blocker. Ginkgo improved memory retention by enhancing the glutamatergic system partially [75].

Panax ginseng C.A. Meyer (Araliaceae): Ginsenosides Rg3(R), $\operatorname{Rg} 3(S)$ or $\operatorname{Rg} 5 / \operatorname{Rk} 1$ (a mixture of $\operatorname{Rg} 5$ and Rk1, $1: 1$. w/w) significantly ameliorated ethanol-induced memory impairment. In addition, ginsenosides $\operatorname{Rg} 3(\mathrm{~S})$ and $\mathrm{Rg} 5 / \mathrm{Rk} 1$ significantly alleviated scopolamine-induced memory impairment. Collectively, neuroprotective actions of these three ginsenosides against memory impairment may be partly attributed to their anti-excitotoxic (glutamate or $N$-methyl-D-aspartate) abilities [76]. Ginseng has 
long been used as a tonic remedy and is one of the most widely used medicinal plants for memory enhancement or anti-aging. The memory enhancing effects of two representative constituents in ginseng, ginsenoside $\mathrm{Rb} 1$ and $\mathrm{Rg} 1$ have been widely studied while the roles of other ginsenosides in anti-amnesia were unclear. To the best of our knowledge, that was the first research paper published in English demonstrating the memory enhancing effects of ginsenosides Rg3(S) and Rg5/Rk1.

Uncariae rhynchophylla (Miq.) Jacks. (Rubiaceae): Its methanolic extract or alkaloid fraction has been shown to reduce ethanol-induced amnesia by recovering an ethanol-induced decrease in glutamic acid level [77].

\section{Action through serotonergic nervous system}

Serotonin (5-HT) has a dual role in the cognitive process that can either strengthen or suppress memory depending on the timing of memory formation. Among the different subtypes of 5HT receptors, 5- $\mathrm{HT}_{1 \mathrm{~A}}, 5-\mathrm{HT}_{4}, 5-\mathrm{HT}_{6}$ and/or $5-\mathrm{HT}_{7}$ receptors play prominent roles in memory formation. The $5-\mathrm{HT}_{3}$ receptor antagonism approach has been proposed for treatment of amnesia. Release of $\mathrm{ACh}$ in the cortex was inhibitorily controlled by the $5-\mathrm{HT}_{3}$ receptor [78].

Schisandra chinensis (Turcz.) Baill. (Tremellaceae): The water layer of fruit of $S$. chinensis counteracted the cycloheximide-induced amnesia. The ameliorating effect was amplified by treatment with a serotonergic $5-\mathrm{HT}_{2}$ receptor antagonist (ritanserin) but reduced by treatment with a serotonergic $5-\mathrm{HT}_{1 \mathrm{~A}}$ receptor agonist (8-OH-DPAT) as well as $\mathrm{GABA}_{\mathrm{A}}$ (bicuculline) and cholinergic receptor antagonists (scopolamine) [79].

Gastrodia elata Bl. (Orchidaceae): p-Hydroxybenzyl alcohol (HBA) (22) ( Fig. 1) is an aglycone of gastrodin and active ingredient of $G$. elata. Results showed that HBA can attenuate $p$-chloroamphetamine (serotonin releaser) or apomorphine (dopaminergic receptor agonist) induced cognitive impairments but not the scopolamine induced ones. These findings suggested that the cognitive enhancing effect of HBA may be due to the suppression of dopaminergic and serotonergic activities [80]. HBA was reported to counteract the cycloheximide-induced shortening of retention latencies. The beneficial effect of HBA on cycloheximide-induced impairment was amplified by treatment with serotonergic receptor antagonists but reduced by serotonergic 5$\mathrm{HT}_{1 \mathrm{~A}}$ and $5 \mathrm{HT}_{2}$ receptor agonists [81].

Hypericum perforatum $\mathrm{L}$. (Guttiferae): Its ethanolic extract reversed scopolamine-induced amnesia with involvement of adrenergic and serotonergic $5-\mathrm{HT}_{1 \mathrm{~A}}$ receptors for retrieval memory. The protective effect conferred by $H$. perforatum was abolished in the presence of serotonergic $5-\mathrm{HT}_{1 \mathrm{~A}}$ receptor antagonist (-)-pindolol, alpha adrenoceptor antagonist phentolamine or beta receptor antagonist propranolol [82].

Polygonatum multiflorum Thunb. (Polygonacease): The cycloheximide-induced memory consolidation impairment was reversed by emodin (23) ( Fig. 1) which can be isolated from the dried root of $P$. multiflorum (Polygonaceae). The beneficial effects were amplified by serotonergic $5-\mathrm{HT}_{1 \mathrm{~A}}$ receptor partial agonist and $5-\mathrm{HT}_{2}$ receptor antagonist but reduced by muscarinic receptor antagonist [83].

Uncariae rhynchophylla (Miq.) Jacks. (Rubiaceae): Its methanolic extract or alkaloid fraction has been shown to reduce ethanol-induced amnesia by recovering ethanol-induced decrease in 5-hydroxytryptamine [77].

\section{Action through other nervous systems}

Ginkgo biloba L. (Ginkgoaceae): Standardized GBE was reported to antagonize scopolamine-induced spatial memory deficit [84]. It was found to inhibit a diphenhydramine- (potent anticholinergic agent)-induced or pyrilamine $\left(\mathrm{H}_{1}\right.$ receptor antagonist)-induced increase in the total error, reference working error and working memory error. These findings concluded that standardized GBE confers protection partially through cholinergic and histaminergic mechanisms. Histamine has been reported to play an important role in learning and memory via $\mathrm{H} 1$ receptors [85, 86].

Panax ginseng C.A. Mey. (Araliaceae): Ethanol-induced acquisition impairment was significantly reduced by single or repeated administration of red ginseng total saponins (RGTS). The inhibitory effect of RGTS on ethanol-induced amnesia was abolished by pretreatment of alpha-methyl-p-tyrosine (inhibitor of catecholamine synthesis) in a dose-response manner but not by $p$ chlorophenylalanine (inhibitor of serotonin synthesis). RGTS confer anti-amnesic effects through catecholaminergic rather than serotonergic neuronal activity [87].

\section{Three Herbs Showing Promising Memory Enhancing Capabilities \\ $\nabla$}

Extensive basic research studies on amnesia have been conducted over the past few decades with the attempt to investigate the anti-amnesic therapeutic potentials of various herbs. Although a number of herbal medicines have been demonstrated with anti-amnesic effects, clinical trials on the use of herbal medicines for the treatment of amnesia have not yet been published. Despite the difficulty of new drug development, several research groups have discovered some herbs with promising efficacies in the clinical setting and great application potential in memory enhancement in healthy subjects, patients with dementia or $\mathrm{AD}$ which could be potential candidates for amnesia as well. Clinical data regarding trials of Centella asiatica, G. biloba and $H$. serrata in human beings are summarized below.

\section{Centella asiatica (L.) Urb., (Apiaceae)}

The cognitive-enhancing effect of C. asiatica (250, 500 and $750 \mathrm{mg}$ once daily for 2 months) was tested in 28 healthy elderly subjects in a randomized, placebo-controlled, double-blind study. Treatment with a high dose $(750 \mathrm{mg})$ of $C$. asiatica repeatedly for two months increased the percentage accuracy of both numeric working memory and word recognition, the reaction time of both numeric working memory and spatial memory as well as the N100 component amplitude of event-related potential [88].

\section{Ginkgo biloba L. (Ginkgoaceae)}

In a randomized, double-blind, placebo-controlled trial, healthy volunteered participants were randomly divided into two groups: Ginkgo (40 mg, thrice/day) and matching placebo for 6 weeks. There was no significant difference in standard neuropsychological tests of learning, memory, attention and concentration or naming and verbal fluency between the ginkgo and placebo groups [89]. In another randomized, double-blind, placebocontrolled clinical trial, 3069 volunteers were assessed every 6 months for incident dementia with a median follow-up of 6.1 years. The overall dementia rate was 3.3 per 100 person years in Ginkgo group versus 2.9 per 100 person years in placebo group. It 
was concluded that G. biloba at $120 \mathrm{mg}$ twice a day did not reduce the occurrence of dementia or $\mathrm{AD}$ in elderly people with normal cognition or with mild cognitive impairment [90].

\section{Huperzia serrata (Thunb.) Trev. (Lycopodiaceae)}

Huperzine A, derived from $\mathrm{H}$. serrata, has been shown to have antioxidant and neuroprotective functions. The neuroprotective effects of huperzine A have been widely studied in AD patients especially in China. It has been suggested that this herb may be as effective as tacrine and donepezil in the symptomatic treatment of dementia. Clinical efficacy and safety of huperzine A has been demonstrated in $A D$ patients in different randomized and placebo-controlled trials in China. In an earlier study, fifty AD patients were administrated with either huperzine $A$ or placebo for eight weeks. Improvement in memory, cognition and behavioral functions was shown in $58 \%$ of the huperzine A-treated group and only $36 \%$ of the placebo group [91]. In another larger scale clinical study, 202 patients aged between 50 and 80 were divided into two groups: Huperzine $A(n=100,100 \mu g$ twice for week 1 ; $150 \mu \mathrm{g}$ twice for week $2-3 ; 200 \mu \mathrm{g}$ twice for week $4-12$ ) or placebo for 12 weeks. Improvement in terms of cognition, behavior, mood and activity of daily life was found in $70 \%$ of the huperzine A-treated groups but only $36 \%$ of the placebo group [92]. Another study has also demonstrated that huperzine A is 8-fold more potent than donepezil and 2-fold more potent than rivastigmine in increasing cortical Ach levels and has a longer lasting effect [93].

\section{Difficulties Hindering Transition to Clinical Studies \\ $\nabla$}

One of the obstacles hindering the transition of basic animal research to human clinical research may be attributed to the dispersed and unsystematic basic experimental design. Most of the studies included a sham or placebo control, but not a positive control. It would have been valuable to compare the efficacy of these herbal medicines with the commonly used FDA-approved AChE inhibitors like tacrine (available since 1993), donepezil (available since 1996), rivastigmine (available since 2000) and galantamine (approved in 2001). Besides, most of the aforementioned animal studies only examine one mechanism of the antiamnesic effect while the lack of follow-up studies on other relevant pathways prevents further progress on understanding the comprehensive effect of these herbs on amnesia.

The anti-amnesic effect of herbal medicine may stem from protective actions on the cholinergic, GABAergic, glutamatergic, serotonergic, catecholaminergic and histaminergic nervous systems. Protective actions on the dopaminergic system cannot be neglected as dopaminergic agents have been reported to ameliorate anoxia-induced memory impairments and enhance learning in healthy adults $[94,95]$. The cAMP-PKA signaling pathway has been suggested to modulate dopaminergic neurotransmission of synaptic plasticity in memory consolidation $[96,97]$. However, the mechanisms of the regulation of synaptic plasticity by neurotransmitters and intracellular signal transduction are not yet fully elucidated. Given the scant information on the underlying anti-amnesia mechanisms, how these herbal medicines confer protection will definitely require further investigation and elucidation.

In new drug development, data obtained from animal studies provide a framework for further clinical trials. Appropriate translation of drug dose across species would definitely have a great impact on the overall effectiveness. By the conventional transla- tion method, animal dose multiplied by human body weight $(60 \mathrm{~kg})$ equal to human equivalent dose, could cause serious misinterpretation and inapplicability of the research [98]. Instead, another calculation, the body surface area (BSA) normalization method which considers several parameters like oxygen utilization, caloric expenditure, basal metabolism, blood volume, circulating plasma proteins and renal functions could provide a more appropriate conversion of drug doses from animal studies to human trials [99]. The formula for dose translation based on BSA is as follow:

\section{Human equivalent dose $(\mathrm{mg} / \mathrm{kg})=$ Animal dose $(\mathrm{mg} / \mathrm{kg}) \times($ Animal $\mathrm{Km} /$ Human $\mathrm{Km}$ )}

where $\mathrm{Km}$ factor is equal to body weight $(\mathrm{kg}) / \mathrm{BSA}\left(\mathrm{m}^{2}\right)$. However, BSA dosing does not take the complex process of drug elimination into consideration therefore overdosing and underdosing could occur affecting the overall therapeutic effects. Further research effort is still needed to formulate a better dose translation method across different species.

\section{Characteristics of Herbs Suitable for Treating Amnesia $\nabla$}

There are three critical criteria for selecting herbal drugs for the treatment of amnesia, including (1) high bioavailability, (2) ability to cross the BBB and (3) minimal adverse and toxic effects.

Bioavailability is defined as the "absorption and utilization of a nutrient" [100]. Upon oral consumption, a herbal extract is expected to cross the intestinal barrier and enter the systemic circulation. The bioavailability of herbs can vary considerably from different plant types and from person-to-person. Based on the studies from Manach et al. and Williamson and Manach in 2005 $[101,102]$, the bioavailability of different types of flavonoids is as follows (from most to least bioavailable): isoflavones, gallic acid > catechins, flavones, quercetin glucosides > proanthocyanidins, galloylated tea catechins, anthocyanins. Future research on pharmacokinetics and pharmacodynamics studies of other bioactive components could be beneficial to new drug development.

For any drugs to be effectively functional in the CNS, they must first penetrate through the BBB. Numerous efflux transporters are expressed on the surface of BBB, such as P-glycoprotein, multi-drug resistance associated protein and monocarboxylic acid transporters. Flavonoids interact with one or several of these transporters either directly or indirectly through stimulatory or inhibitory modes [103]. Two bioactives in Ginkgo, quercetin and kaempferol stimulate P-glycoprotein transporters whereas resveratrol in grape seeds inhibit P-glycoprotein transporters. The bioavailability of herbal drugs for the treatment of amnesia depends greatly on the extent to which they can cross the BBB.

While most of the available literature has been concentrated on studying efficacies of various herbs, information concerning the toxicological studies of herbal treatments on amnesia has been scanted so far. There were neither reports of any serious adverse nor toxic effects for the aforementioned herbs. To ensure the safe uses of these plant extracts or their bioactive components, acute and chronic toxicity tests in animals would be helpful to identify any potential hazards for human consumption although the aforementioned herbs do not classify as toxic herbs with reference to the Pharmacopoeia of People's Republic of China. Besides, dosages of herbal medicines, like other Western pharmaceutics, play an important role in the overall pharmacological and toxic 
effects. In developing therapeutic strategies for treating amnesia, further investigation on bioavailability, BBB penetration and toxic/adverse effects would be invaluable.

\section{Current Issues and Future Perspectives $\nabla$}

Administration of medication to regulate the neurotransmitters is one of the methods for the treatment of amnesia. Currently, the use of AChE inhibitors to treat amnesia is still the mainstream pharmacotherapy. Although cholinesterase inhibitors - donepezil, galantamine, rivastigmine and tacrine are the four approved pharmacological therapies for dementia by the U.S. Food and Drug Administration, Raina et al. indicated that cholinesterase inhibitors only resulted in marginal clinical improvement as measured by cognition and global assessments [104].

\section{Rapid screening techniques}

Numerous activities of different active ingredients in herbs may be relevant to the treatment of cognitive disorders while the complicated pathophysiology of amnesia has not been fully elucidated. With special attention paid to the enhancement of cholinergic neurotransmission, the majority of research has been focused on anti-cholinesterase alkaloids. However, other pharmacological targets are also of great importance. Given a diverse array of compounds from thousands of herbs, a rapid screening method would be useful to delineate the scope of potential compounds for in-depth investigation. cDNA microarray technology which simultaneously monitors several pathways could possibly accelerate the elucidation of the underlying mechanisms of potential herbal medicines and provide solid evidence for further clinical investigation. With this new methodology, the overall cellular changes and wide spectrum of differentially expressed genes upon chemical-induced amnesia can be revealed. Alternative novel pathways such as apoptosis, cytoskeleton reconstruction, protein trafficking and cell differentiation can be closely monitored in addition to targeting muscarinic ACh receptors and their associated signaling molecules [105]. Recently, using the microarray and quantitative real-time RT-PCR approaches, Homer1, GABA(B) receptor, early growth response 1, prodynorphin, VGF nerve growth factor inducible and several novel genes including calcium/calmodulin-dependent protein kinase 2 and glycophorin $\mathrm{C}$ were revealed following scopolamine treatment [106]. The above proteins and RNAs could be targeted and modulated by the administration of herbal medicines that may have beneficial effects on amnesia.

\section{Genetically knockout mice models}

As the pathophysiology of amnesia is complicated where different mechanistic pathways are interrelated, the uses of genetic knockout mice models which are deficient in particular receptors of neurotransmitters or specific antioxidation relevant genes may help to delineate protective effects of herbs or active therapeutic components. Most of the aforementioned reports are unable to conclude whether the observed improvement was due to a single direct action of the drug at, for example, the cholinergic system, or the combinative actions at several different sites. Employing genetically knockout mice to study anti-amnesic effects of potential herbs will give a clearer picture of at least some of the underlying mechanisms.
Synthetic single compound with multiple target actions A herbal medicine, which shows favorable effects on cognitive disorders as evident in a wide variety of experimental studies, has a great potential for clinical use. Active ingredients in herbal medicine can act synergistically with other ingredients as well as counterbalance toxic effects of other active components. In 2007, Decker proposed the idea of hybrid molecules in which a single molecule has multiple pharmacological actions [107]. Administration of a single compound is expected to result in more predictable pharmacokinetics and pharmacodynamics as well as improved compliance in patients. One part of the hybrid molecule can have AChE inhibitory activity whereas another part can target GABAergic, glutaminergic, histaminergic, serotonergic and catecholaminergic systems. Achieving an optimal balance of the therapeutic activities of the different parts of the hybrid molecules is more important than obtaining the maximal activity of a single part.

The degree of recovery and the duration of treatment period using herbal medicine are expected to vary with the location and severity of the lesioned brain areas as well as the type of amnesia. Different types of herbs may also be beneficial for different damaged brain areas or types of amnesia. Further in-depth research would be valuable to all amnesic patients.

\section{Conclusion}

$\nabla$

Many herbs have been preliminarily demonstrated to pose protective effects against amnesia in animal models. Natural products not only demonstrate specific effects on particular pathways, but also act synergistically on the neural systems with other components. However, limited studies on the mechanism, pharmacology and toxicology of these herbal medicines are available, which seriously hinder their development for clinical uses. It will further be of value to elucidate the structural features and bioactives of herbal drugs that are relevant to their neuroprotective properties. In addition to current cholinergic-based strategies, other approaches involving GABAergic, glutaminergic, catecholaminergic, histaminergic, serotonergic and dopaminergic systems are also critical to rationalize drug development for the treatment of amnesia, dementia and other related neurological complications.

\section{References}

1 Eysenck MW, Keane MT. Cognitive psychology: a student's handbook. New York: Psychology Press; 2005

2 Cheng Y, Shen LH, Zhang JT. Anti-amnestic and anti-aging effects of ginsenoside Rg1 and Rb1 and its mechanism of action. Acta Pharmacol Sin 2005; 26: 143-149

3 Kennedy DO, Scholey $A B$. Ginseng: potential for the enhancement of cognitive performance and mood. Pharmacol Biochem Behav 2003; 75: 687-700

4 Nishijo H, Uwano T, Zhong YM, Ono T. Proof of the mysterious efficacy of ginseng: basic and clinical trials: effects of red ginseng on learning and memory deficits in an animal model of amnesia. J Pharmacol Sci 2004; 95: $145-152$

5 Blumenthal M, Busse WR, Bundesinstitut für Arzneimittel und Medizinprodukte. The complete German Commission E monographs: therapeutic guide to herbal medicines. Austin: American Botanical Council; 1998

6 Heinrich M, Lee TH. Galanthamine from snowdrop - the development of a modern drug against Alzheimer's disease from local Caucasian knowledge. J Ethnopharmacol 2004; 92: 147-162

7 Erkinjuntti T, Kurz A, Gauthier S, Bullock R, Lilienfeld S, Damaraju CV. Efficacy of galantamine in probable vascular dementia and Alzheimer's 
disease combined with cerebrovascular disease: a randomised trial. Lancet 2002; 359: 1283-1290

8 Brown TH, Chapman PF, Kairiss EW, Keenan CL. Long-term synaptic potentiation. Science 1988; 242: 724-728

9 Centonze D, Picconi B, Gubellini P, Bernardi G, Calabresi P. Dopaminergic control of synaptic plasticity in the dorsal striatum. Eur J Neurosci 2001; 13: 1071-1077

10 Munro CA, Walling SG, Evans JH, Harley CW. Beta-adrenergic blockade in the dentate gyrus in vivo prevents high frequency-induced longterm potentiation of EPSP slope, but not long-term potentiation of population spike amplitude. Hippocampus 2001; 11: 322-328

11 Ohashi S, Matsumoto M, Otani H, Mori K, Togashi H, Ueno K, Kaku A, Yoshioka $\mathrm{M}$. Changes in synaptic plasticity in the rat hippocampo-medial prefrontal cortex pathway induced by repeated treatments with fluvoxamine. Brain Res 2002; 949: 131-138

12 Yamazaki Y, Hamaue N, Sumikawa K. Nicotine compensates for the loss of cholinergic function to enhance long-term potentiation induction. Brain Res 2002; 946: 148-152

13 Myhrer T. Neurotransmitter systems involved in learning and memory in the rat: a meta-analysis based on studies of four behavioral tasks. Brain Res Brain Res Rev 2003; 41: 268-287

14 Quartermain $D$, Leo $P$. Strength of scopolamine-induced amnesia as a function of time between training and testing. Behav Neural Biol 1988; 50: 300-310

15 Hatakeyama D, Sadamoto H, Watanabe T, Wagatsuma A, Kobayashi S, Fujito Y, Yamashita $M$, Sakakibara $M$, Kemenes $G$, Ito E. Requirement of new protein synthesis of a transcription factor for memory consolidation: paradoxical changes in mRNA and protein levels of C/EBP. J Mol Biol 2006; 356: 569-577

16 Barraco RA, Stettner LJ. Antibiotics and memory. Psychol Bull 1976; 83: 242-302

17 Davis HP, Squire LR. Protein synthesis and memory: a review. Psychol Bull 1984; 96: 518-559

18 Ichihara K, Nabeshima T, Kameyama T. Opposite effects induced by low and high doses of apomorphine on single-trial passive avoidance learning in mice. Pharmacol Biochem Behav 1988; 30: 107-113

19 Ogren SO, Johansson C. Separation of the associative and non-associative effects of brain serotonin released by $p$-chloroamphetamine: dissociable serotoninergic involvement in avoidance learning, pain and motor function. Psychopharmacology (Berlin) 1985; 86: 12-26

20 Levin ED, Briggs SJ, Christopher NC, Auman JT. Working memory performance and cholinergic effects in the ventral tegmental area and substantia nigra. Brain Res 1994; 657: 165-170

21 Nooshinfar E, Lashgari R, Haghparast A, Sajjadi S. NMDA receptors are involved in Ginkgo extract-induced facilitation on memory retention of passive avoidance learning in rats. Neurosci Lett 2008; 432: 206-211

22 Hagan JJ, Salamone JD, Simpson J, Iversen SD, Morris RG. Place navigation in rats is impaired by lesions of medial septum and diagonal band but not nucleus basalis magnocellularis. Behav Brain Res 1988; 27: 920

23 Hodges H, Allen Y, Kershaw T, Lantos PL, Gray JA, Sinden J. Effects of cholinergic-rich neural grafts on radial maze performance of rats after excitotoxic lesions of the forebrain cholinergic projection system - I. Amelioration of cognitive deficits by transplants into cortex and hippocampus but not into basal forebrain. Neuroscience 1991; 45: 587-607

24 Wenk GL, Cribbs B, McCall L. Nucleus basalis magnocellularis: optimal coordinates for selective reduction of choline acetyltransferase in frontal neocortex by ibotenic acid injections. Exp Brain Res 1984; 56: 335340

25 Hasselmo ME, Giocomo LM. Cholinergic modulation of cortical function. J Mol Neurosci 2006; 30: 133-135

26 Doods HN, Quirion R, Mihm G, Engel W, Rudolf K, Entzeroth M, Schiavi GB, Ladinsky H, Bechtel WD, Ensinger HA. Therapeutic potential of CNS-active M2 antagonists: novel structures and pharmacology. Life Sci 1993; 52: 497-503

27 Hammel P, Larrey D, Bernuau J, Kalafat M, Freneaux E, Babany G, Degott C, Feldmann G, Pessayre D, Benhamou JP. Acute hepatitis after tetrahydroaminoacridine administration for Alzheimer's disease. J Clin Gastroenterol 1990; 12: 329-331

28 Watkins PB, Zimmerman HJ, Knapp MJ, Gracon SI, Lewis KW. Hepatotoxic effects of tacrine administration in patients with Alzheimer's disease. JAMA 1994; 271: 992-998

29 Ohta H, Ni JW, Matsumoto K, Watanabe H, Shimizu M. Peony and its major constituent, paeoniflorin, improve radial maze performance im- paired by scopolamine in rats. Pharmacol Biochem Behav 1993; 45: 719-723

30 Kang SY, Lee KY, Park MJ, Kim YC, Markelonis GJ, Oh TH. Decursin from Angelica gigas mitigates amnesia induced by scopolamine in mice. Neurobiol Learn Mem 2003; 79: 11-18

31 Kim DH, Kim DY, Kim YC, Jung JW, Lee S, Yoon BH, Cheong JH, Kim YS, Kang SS, Ko KH. Nodakenin, a coumarin compound, ameliorates scopolamine-induced memory disruption in mice. Life Sci 2007; 80: 19441950

32 Murakami A, Gao G, Kim OK, Omura M, Yano M, Ito C, Furukawa H, Jiwajinda S, Koshimizu K, Ohigashi H. Identification of coumarins from the fruit of Citrus hystrix DC as inhibitors of nitric oxide generation in mouse macrophage RAW 264.7 cells. J Agric Food Chem 1999; 47: 333-339

33 Cho J, Yang CH, Park CG, Lee H, Kim YH. Inhibition of excitotoxic neuronal cell death by total extracts from oriental medicines used for stroke treatment. Yakhak Hoeji 2000; 44: 29-35

34 Lee B, Choi Y, Kim H, Kim SY, Hahm DH, Lee HJ, Shim I. Protective effects of methanol extract of Acori graminei rhizoma and Uncariae Ramulus et Uncus on ischemia-induced neuronal death and cognitive impairments in the rat. Life Sci 2003; 74: 435-450

35 Irie $Y$, Keung WM. Rhizoma acori graminei and its active principles protect PC-12 cells from the toxic effect of amyloid-beta peptide. Brain Res 2003; 963: 282-289

$36 \mathrm{Kim} \mathrm{JH,} \mathrm{Hahm} \mathrm{DH,} \mathrm{Lee} \mathrm{HJ,} \mathrm{Pyun} \mathrm{KH,} \mathrm{Shim} \mathrm{I.} \mathrm{Acori} \mathrm{graminei} \mathrm{rhizoma} \mathrm{ame-}$ liorated ibotenic acid-induced amnesia in rats. Evid Based Complement Alternat Med 2009; 6; 457-464 (DOI: 10.1093/ecam/nem158)

37 Hsieh MT, Wu CR, Lin LW, Hsieh CC, Tsai CH. Reversal caused by n-butylidenephthalide from the deficits of inhibitory avoidance performance in rats. Planta Med 2001; 67: 38-42

38 Hsieh MT, Tsai FH, Lin YC, Wang WH, Wu CR. Effects of ferulic acid on the impairment of inhibitory avoidance performance in rats. Planta Med 2002; 68: 754-756

39 Peng WH, Hsieh MT, Wu CR. Effect of long-term administration of berberine on scopolamine-induced amnesia in rats. Jpn J Pharmacol 1997; 74: $261-266$

40 Hung TM, Ngoc TM, Youn UJ, Min BS, Na M, Thuong PT, Bae K. Anti-amnestic activity of pseudocoptisine from Corydalis tuber. Biol Pharm Bull 2008; 31: 159-162

41 Hsieh MT, Hsieh CL, Wang WH, Chen CS, Lin CJ, Wu CR. Osthole improves aspects of spatial performance in ovariectomized rats. Am J Chin Med 2004; $32: 11-20$

42 Joshi H, Parle M. Antiamnesic effects of Desmodium gangeticum in mice. Yakugaku Zasshi 2006; 126: 795-804

43 Joshi $H$, Parle M. Cholinergic basis of memory-strengthening effect of Foeniculum vulgare Linn. J Med Food 2006; 9: 413-417

44 Lima JA, Costa RS, Epifanio RA, Castro NG, Rocha MS, Pinto AC. Geissospermum vellosii stembark: anticholinesterase activity and improvement of scopolamine-induced memory deficits. Pharmacol Biochem Behav 2009; 92: 508-513

45 Das A, Shanker G, Nath C, Pal R, Singh S, Singh H. A comparative study in rodents of standardized extracts of Bacopa monniera and Ginkgo bilo$b a$ : anticholinesterase and cognitive enhancing activities. Pharmacol Biochem Behav 2002: 73: 893-900

46 Cheng DH, Ren H, Tang XC. Huperzine A, a novel promising acetylcholinesterase inhibitor. Neuroreport 1996; 8: 97-101

47 Wu CR, Lin LW, Wang WH, Hsieh MT. The ameliorating effects of LiuWei Dihuang Wang on cycloheximide-induced impairment of passive avoidance performance in rats. J Ethnopharmacol 2007; 113: 79-84

48 Hsieh MT, Cheng SJ, Lin LW, Wang WH, Wu CR. The ameliorating effects of acute and chronic administration of LiuWei Dihuang Wang on learning performance in rodents. Biol Pharm Bull 2003; 26: 156-161

49 Tsai FS, Peng WH, Wang WH, Wu CR, Hsieh CC, Lin YT, Feng IC, Hsieh MT. Effects of luteolin on learning acquisition in rats: involvement of the central cholinergic system. Life Sci 2007; 80: 1692-1698

50 Liu R, Gao M, Oiang GF, Zhang TT, Lan X, Ying J, Du GH. The anti-amnesic effects of luteolin against amyloid beta(25-35) peptide-induced toxicity in mice involve the protection of neurovascular unit. Neuroscience 2009; 162: 1232-1243

51 Vasudevan M, Parle M. Antiamnesic potential of Murraya koenigii leaves. Phytother Res 2009; 23: 308-316

52 Joshi $H$, Parle M. Nardostachys jatamansi improves learning and memory in mice. J Med Food 2006; 9: 113-118

53 Oh JH, Choi BJ, Chang MS, Park SK. Nelumbo nucifera semen extract improves memory in rats with scopolamine-induced amnesia through 
the induction of choline acetyltransferase expression. Neurosci Lett 2009; 461: 41-44

54 Zhang DS, Zhang JT. Effect of total ginsenoside on synaptic transmission in dentate gyrus in rats. Acta Pharmacol Sin 2000; 35: 185-188

55 Wang XY, Zhang JT. Effects of ginsenoside Rg1 on synaptic plasticity of freely moving rats and its mechanism of action. Acta Pharmacol Sin 2001; 22: 657-662

56 Zhang JT, Liu Y, Qu ZW, Zhang XL, Xiao HL. Influence of ginsenoside Rb1 and Rg1 on some central neurotransmitter receptors and protein biosynthesis in the mouse brain. Yao Xue Xue Bao 1988; 23: 12-16

57 Zhang JT, Qu ZW, Liu Y, Deng HL. Preliminary study on antiamnestic mechanism of ginsenoside Rg1 and Rb1. Chin Med J (Engl) 1990; 103: 932-938

58 Jin SH, Park JK, Nam KY, Park SN, Jung NP. Korean red ginseng saponins with low ratios of protopanaxadiol and protopanaxatriol saponin improve scopolamine-induced learning disability and spatial working memory in mice. J Ethnopharmacol 1999; 66: 123-129

59 Ikeya Y, Takeda S, Tunakawa M, Karakida H, Toda K, Yamaguchi T, Aburada $M$. Cognitive improving and cerebral protective effects of acylated oligosaccharides in Polygala tenuifolia. Biol Pharm Bull 2004; 27: 1081-1085

60 Hsieh MT, Kuo LH, Tsai FH, Wang WH, Wu CR. Effects of puerarin on scopolamine-, mecamylamine-, p-chloroamphetamine- and dizocilpineinduced inhibitory avoidance performance impairment in rats. Planta Med 2002; 68: 901-905

61 Heo HJ, Suh YM, Kim MJ, Choi SJ, Mun NS, Kim HK, Kim E, Kim CJ, Cho HY, Kim YJ, Shin DH. Daidzein activates choline acetyltransferase from MC-IXC cells and improves drug-induced amnesia. Biosci Biotechnol Biochem 2006; 70: 107-111

62 Kim DH, Jeon SJ, Jung JW, Lee S, Yoon BH, Shin BY, Son KH, Cheong JH, Kim YS, Kang SS. Tanshinone congeners improve memory impairments induced by scopolamine on passive avoidance tasks in mice. Eur J Pharmacol 2007; 574: 140-147

63 Orhan I, Aslan M. Appraisal of scopolamine-induced antiamnesic effect in mice and in vitro antiacetylcholinesterase and antioxidant activities of some traditionally used Lamiaceae plants. J Ethnopharmacol 2009; 122: 327-332

64 Jeong EJ, Lee KY, Kim SH, Sung SH, Kim YC. Cognitive-enhancing and antioxidant activities of iridoid glycosides from Scrophularia buergeriana in scopolamine-treated mice. Eur J Pharmacol 2008; 588: 78-84

65 Kim SR, Kang SY, Lee KY, Kim SH, Markelonis GJ, Oh TH, Kim YC. Anti-amnestic activity of E-p-methoxycinnamic acid from Scrophularia buergeriana. Brain Res Cogn Brain Res 2003; 17: 454-461

66 Kim DH, Hung TM, Bae KH, Jung JW, Lee S, Yoon BH, Cheong JH, Ko KH, Ryu JH. Gomisin A improves scopolamine-induced memory impairment in mice. Eur J Pharmacol 2006; 542: 129-135

67 Vasudevan M, Parle M. Pharmacological actions of Thespesia populnea relevant to Alzheimer's disease. Phytomedicine 2006; 13: 677-687

$68 \mathrm{Kim} \mathrm{JH}, \mathrm{Ha} \mathrm{HC}$, Lee MS, Kang JI, Kim HS, Lee SY, Pyun KH, Shim I. Effect of Tremella fuciformis on the neurite outgrowth of PC12 h cells and the improvement of memory in rats. Biol Pharm Bull 2007; 30: 708-714

69 Kim JH, Chung JY, Lee YJ, Park S, Hahm DH, Lee HJ, Shim I. Effects of methanol extract of Uncariae Ramulus et Uncus on ibotenic acid-induced amnesia in the rat. J Pharmacol Sci 2004; 96: 314-323

70 Prabhakar S, Saraf MK, Pandhi P, Anand A. Bacopa monniera exerts antiamnesic effect on diazepam-induced anterograde amnesia in mice. Psychopharmacology (Berlin) 2008; 200: 27-37

71 Kim DH, Jeon SJ, Son KH, Jung JW, Lee S, Yoon BH, Lee JJ, Cho YW, Cheong $\mathrm{JH}$, Ko KH, Ryu JH. The ameliorating effect of oroxylin A on scopolamine-induced memory impairment in mice. Neurobiol Learn Mem 2007; 87: 536-546

72 Shors TJ, Servatius RJ, Thompson RF, Rogers G, Lynch G. Enhanced glutamatergic neurotransmission facilitates classical conditioning in the freely moving rat. Neurosci Lett 1995; 186: 153-156

73 Lynch G, Granger R, Ambros-Ingerson J, Davis CM, Kessler M, Schehr R. Evidence that a positive modulator of AMPA-type glutamate receptors improves delayed recall in aged humans. Exp Neurol 1997; 145: 89-92

74 Collingridge GL, Kehl SJ, McLennan H. Excitatory amino acids in synaptic transmission in the Schaffer collateral-commissural pathway of the rat hippocampus. J Physiol 1983; 334: 33-46

75 Nooshinfar E, Lashgari R, Haghparast A, Sajjadi S. NMDA receptors are involved in Ginkgo extract-induced facilitation on memory retention of passive avoidance learning in rats. Neurosci Lett 2008; 432: 206-211
76 Bao HY, Zhang J, Yeo SJ, Myung CS, Kim HM, Kim JM, Park JH, Cho J, Kang $J S$. Memory enhancing and neuroprotective effects of selected ginsenosides. Arch Pharm Res 2005; 28: 335-342

77 Lee SC, Linh PT, Jing Z, Ryu SY, Myung CS, Kim YH, Kang JS. Effects of repeated administration of Uncaria hooks on the acquisition and central neuronal activities in ethanol-treated mice. J Ethnopharmacol 2004; 94: $123-128$

78 Barnes NM, Costall B, Naylor RJ, Williams TJ, Wischik CM. Normal densities of 5-HT3 receptor recognition sites in Alzheimer's disease. Neuroreport 1990; $1: 253-254$

79 Hsieh MT, Wu CR, Wang WH, Lin LW. The ameliorating effect of the water layer of Fructus Schisandrae on cycloheximide-induced amnesia in rats: interaction with drugs acting at neurotransmitter receptors. Pharmacol Res 2001; 43: 17-22

80 Wu CR, Hsieh MT, Liao J. p-Hydroxybenzyl alcohol attenuates learning deficits in the inhibitory avoidance task: involvement of serotonergic and dopaminergic systems. Chin J Physiol 1996; 39: 265-273

81 Hsieh MT, Wu CR, Hsieh CC. Ameliorating effect of p-hydroxybenzyl alcohol on cycloheximide-induced impairment of passive avoidance response in rats: interactions with compounds acting at 5-HT1A and 5HT2 receptors. Pharmacol Biochem Behav 1998; 60: 337-343

82 Khalifa AE. Hypericum perforatum as a nootropic drug: enhancement of retrieval memory of a passive avoidance conditioning paradigm in mice. J Ethnopharmacol 2001; 76: 49-57

83 Lu MC, Hsieh MT, Wu CR, Cheng HY, Hsieh CC, Lin YT, Peng WH. Ameliorating effect of emodin, a constitute of Polygonatum multiflorum, on cycloheximide-induced impairment of memory consolidation in rats. J Ethnopharmacol 2007; 112: 552-556

84 Yamamoto Y, Adachi Y, Fujii Y, Kamei C. Ginkgo biloba extract improves spatial memory in rats mainly but not exclusively via a histaminergic mechanism. Brain Res 2007; 1129: 161-165

85 Kamei C, Tasaka K. Effect of histamine on memory retrieval in old rats. Biol Pharm Bull 1993; 16: 128-132

86 Kamei C, Chung YH, Tasaka K. Influence of certain H1-blockers on the step-through active avoidance response in rats. Psychopharmacology (Berlin) 1990; 102: 312-318

87 Lee SC, Moon YS, You KH. Effects of red ginseng saponins and nootropic drugs on impaired acquisition of ethanol-treated rats in passive avoidance performance. J Ethnopharmacol 2000; 69: 1-8

88 Wattanathorn J, Mator L, Muchimapura S, Tongun T, Pasuriwong O, Piyawatkul N, Yimtae K, Sripanidkulchai B, Singkhoraard J. Positive modulation of cognition and mood in the healthy elderly volunteer following the administration of Centella asiatica. J Ethnopharmacol 2008; 116: 325-332

89 Solomon PR, Adams F, Silver A, Zimmer J, DeVeaux R. Ginkgo for memory enhancement: a randomized controlled trial. JAMA 2002; 288: 835840

90 DeKosky ST, Williamson JD, Fitzpatrick AL, Kronmal RA, Ives DG, Saxton JA, Lopez OL, Burke G, Carlson MC, Fried LP. Ginkgo biloba for prevention of dementia: a randomized controlled trial. JAMA 2008; 300: 22532262

91 Xu SS, Gao ZX, Weng Z, Du ZM, Xu WA, Yang JS, Zhang ML, Tong ZH, Fang $Y S$, Chai XS. Efficacy of tablet huperzine-A on memory, cognition, and behavior in Alzheimer's disease. Zhongguo Yao Li Xue Bao 1995; 16 : 391-395

92 Zhang Z, Wang X, Chen Q, Shu L, Wang J, Shan G. Clinical efficacy and safety of huperzine Alpha in treatment of mild to moderate Alzheimer disease, a placebo-controlled, double-blind, randomized trial. Zhonghua Yi Xue Za Zhi 2002; 82: 941-944

93 Liang YQ Tang XC. Comparative effects of huperzine A, donepezil and rivastigmine on cortical acetylcholine level and acetylcholinesterase activity in rats. Neurosci Lett 2004; 361: 56-59

94 Debette S, Kozlowski O, Steinling M, Rousseaux M. Levodopa and bromocriptine in hypoxic brain injury. J Neurol 2002; 249: 1678-1682

95 Knecht S, Breitenstein C, Bushuven S, Wailke S, Kamping S, Floel A, Zwitserlood P, Ringelstein EB. Levodopa: faster and better word learning in normal humans. Ann Neurol 2004; 56: 20-26

96 Kandel ER. The molecular biology of memory storage: a dialog between genes and synapses. Biosci Rep 2001; 21: 565-611

97 Arnsten AF, Ramos BP, Birnbaum SG, Taylor JR. Protein kinase A as a therapeutic target for memory disorders: rationale and challenges. Trends Mol Med 2005; 11: 121-128 
98 Baur JA, Pearson KJ, Price NL, Jamieson HA, Lerin C, Kalra A, Prabhu VV, Allard JS, Lopez-Lluch G, Lewis K, Pistell PJ, Poosala S, Becker KG, Boss O, Gwinn D, Wang M, Ramaswamy S, Fishbein KW, Spencer RG, Lakatta EG, Le Couteur D, Shaw RJ, Navas P, Puigserver P, Ingram DK, de Cabo $R$, Sinclair DA. Resveratrol improves health and survival of mice on a high-calorie diet. Nature 2006; 444: 337-342

99 Reagan-Shaw S, Nihal M, Ahmad N. Dose translation from animal to human studies revisited. FASEB J 2008; 22: 659-661

100 Krebs NF. Bioavailability of dietary supplements and impact of physiologic state: infants, children and adolescents. J Nutr 2001; 131: 1351S-1354S

101 Manach C, Williamson G, Morand C, Scalbert A, Remesy C. Bioavailability and bioefficacy of polyphenols in humans. I. Review of 97 bioavailability studies. Am J Clin Nutr 2005; 81: 230S-242S

102 Williamson G, Manach C. Bioavailability and bioefficacy of polyphenols in humans. II. Review of 93 intervention studies. Am J Clin Nutr 2005; 81: 243S-255S
103 Youdim KA, Shukitt-Hale B, Joseph JA. Flavonoids and the brain: interactions at the blood-brain barrier and their physiological effects on the central nervous system. Free Radic Biol Med 2004; 37: 1683-1693

104 Raina P, Santaguida P, Ismaila A, Patterson C, Cowan D, Levine M, Booker $L$, Oremus $M$. Effectiveness of cholinesterase inhibitors and memantine for treating dementia: evidence review for a clinical practice guideline. Ann Intern Med 2008; 148: 379-397

105 Hsieh MT, Hsieh CL, Lin LW, Wu CR, Huang GS. Differential gene expression of scopolamine-treated rat hippocampus-application of cDNA microarray technology. Life Sci 2003; 73: 1007-1016

106 Brouillette J, Young D, During MJ, Quirion R. Hippocampal gene expression profiling reveals the possible involvement of Homer1 and GABA (B) receptors in scopolamine-induced amnesia. J Neurochem 2007; 102: 1978-1989

107 Decker M. Recent advances in the development of hybrid molecules/ designed multiple compounds with antiamnesic properties. Mini Rev Med Chem 2007; 7: 221-229 